\title{
The von Neumann Model of Measurement in Quantum Mechanics
}

\author{
Pier A. Mello \\ Instituto de Física, Universidad Nacional Autónoma de México, Apdo. Postal 20-364, 01000 \\ México, D. F., México
}

\begin{abstract}
.
We describe how to obtain information on a quantum-mechanical system by coupling it to a probe and detecting some property of the latter, using a model introduced by von Neumann, which describes the interaction of the system proper with the probe in a dynamical way.

We first discuss single measurements, where the system proper is coupled to one probe with arbitrary coupling strength. The goal is to obtain information on the system detecting the probe position. We find the reduced density operator of the system, and show how Lüders rule emerges as the limiting case of strong coupling.

The von Neumann model is then generalized to two probes that interact successively with the system proper. Now we find information on the system by detecting the position-position and momentum-position correlations of the two probes. The so-called "Wigner's formula" emerges in the strong-coupling limit, while "Kirkwood's quasi-probability distribution" is found as the weakcoupling limit of the above formalism. We show that successive measurements can be used to develop a state-reconstruction scheme.

Finally, we find a generalized transform of the state and the observables based on the notion of successive measurements.
\end{abstract}

PACS: 03.65.Ta, 03.65.Wj

\section{INTRODUCTION}

In a general quantum measurement one obtains information on the system of interest by coupling it to an auxiliary degree of freedom, or probe, and then detecting some property of the latter using a measuring device. This procedure, which was described by von Neumann in his classic book [1], will be referred to as von Neumannn's model (vNM). Within the vNM, the combined system -system proper plus probe- is given a dynamical description.

An example of the idea involved in the vNM is given by the Stern-Gerlach experiment, which is described in every textbooks on QM (see, e.g., [2, 3]).

In this experiment, presented schematically in Fig. 1, the observable we want to obtain information about is the $z$-component of the spin of a particle. The auxiliary degree of freedom, or probe, is its position $\mathbf{r}$-still a microscopic quantity-after it leaves the magnet, and this is what is recorded by a detecting device, which in this case is a position detector. E.g., for $s=1 / 2$, we may wish to find information on the two components $\left\langle\psi_{\text {spin }}\left|\mathbb{P}_{ \pm}^{z}\right| \psi_{\text {spin }}\right\rangle=\left|\left\langle \pm \mid \psi_{\text {spin }}\right\rangle\right|^{2}$ of the original state.

Another example is provided by Cavity Quantum Electrodynamics (QED) experiments $[4,5,6]$. Here the observable is the number $n$ of photons in a cavity. As shown schematically in Fig. 2, the probes are atoms successively sent through the cavity: after 


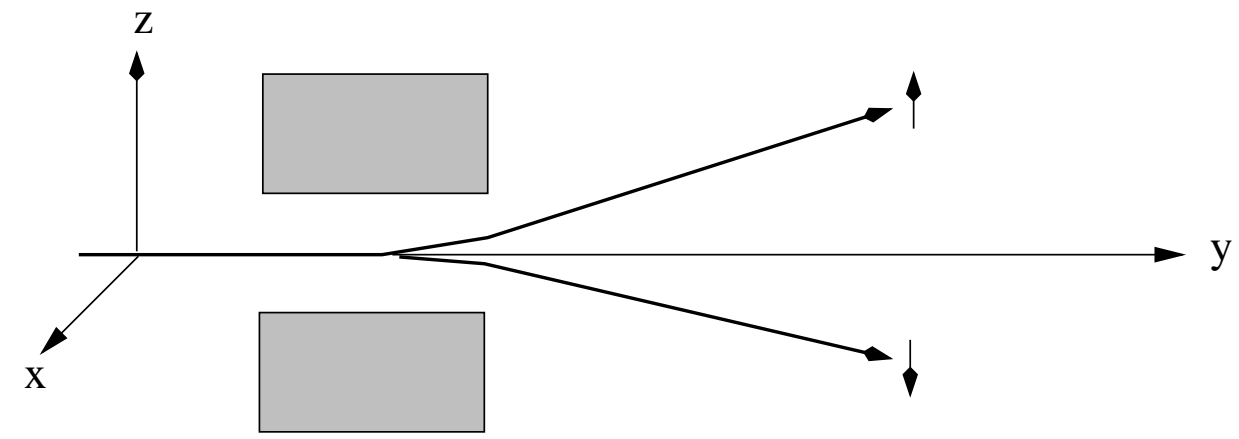

FIGURE 1. The Stern-Gerlach experiment, designed to find information on the $z$-component of the spin of a particle by detecting its position.

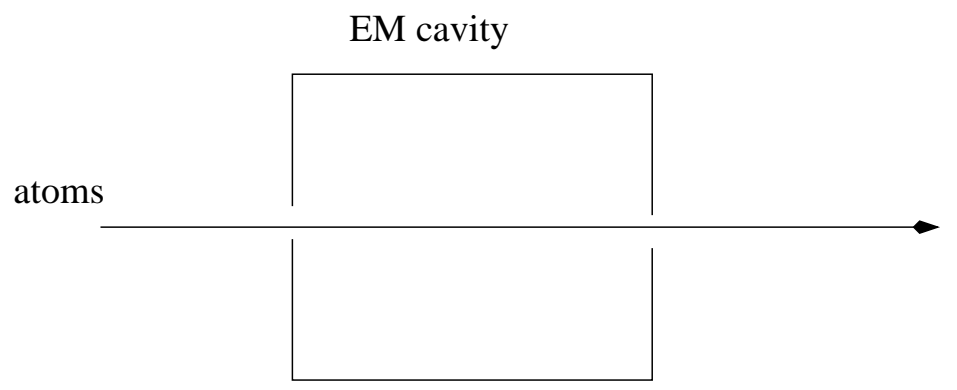

FIGURE 2. A Cavity QED experiment, designed to find information on the number of photons inside a cavity, by sending atoms through the cavity and subsequently detecting them.

they leave the cavity, they are detected by a measuring device.

In what follows we first discuss, in the next section, the Stern-Gerlach problem, which has become a paradigm for models of measurement in QM.

The discussion will pave the way for the analysis, in Sec. 3, of single measurements in $\mathrm{QM}$, where the system proper is coupled to one probe with arbitrary coupling strength. The main goal is to study what information can we obtain on the system by detecting the probe position [7]. As a by-product of the analysis, we obtain the reduced density operator of the system proper after its interaction with the probe, and derive the so-called Lüders rule [8] as the limiting case of strong coupling.

We then generalize the vNM to two probes that interact successively with the system proper [7]. Again, we study what information can we obtain on the system by detecting the position-position and momentum-position correlations of the two probes. Indeed, we describe a state reconstruction scheme based on the procedure of successive measurements $[7,9]$. We obtain the so-called "Wigner's formula" [10] in the strong-coupling limit of the above formalism, and Kirkwood's quasi-probability distribution [11] in the weak-coupling limit. We also find a generalized transform of the state and the observables based on the notion of successive measurements, and in terms of complex quasiprobabilities. 


\section{THE STERN-GERLACH EXPERIMENT}

The Stern-Gerlach experiment, Fig. 1, has become a paradigm for models of measurement in QM. Although the experiment was first performed as early as 1922, it is explained in every textbook on QM (see, e.g. Refs. [2, 3]), and various refinements have been presented in the literature (as in Refs. [12, 13, 14]), surprisingly, its complete (nonrelativistic) solution has been given only recently [15].

By a complete solution we mean one that takes into account the translational and transverse motions of the atom, and a confined magnetic field $\mathbf{B}(\mathbf{r})$ that satisfies Maxwell's equations

$$
\nabla \cdot \mathbf{B}=\mathbf{0}, \quad \nabla \times \mathbf{B}=\mathbf{0} .
$$

Here we shall work with a model of the complete problem which has a simple exact solution and still shows the physical characteristic we want to exhibit, i.e., bending of the trajectory depending on the $z$-projection of the spin.

We shall: i) assume $\mathbf{B} \equiv 0$ outside the gaps; ii) assume that only $B_{z}$ is significant; iii) assume $B_{z}(z) \approx B^{\prime}(z) z$ inside the gaps; iv) simulate the translational motion in the $y$ direction using a $t$-dependent interaction which lasts for the time the particle is inside the gap of the magnet. This could be achieved by adopting a frame of reference moving with the particle; v) ignore the $x$ degree of freedom.

We then consider the model Hamiltonian

$$
\begin{aligned}
\hat{H}(t) & =\frac{\hat{p}_{z}^{2}}{2 m}-\mu \cdot \mathbf{B} \theta_{t_{1}, \tau}(t) \\
& =\frac{\hat{p}_{z}^{2}}{2 m}-\left[\mu_{B} B_{z}^{\prime}(0) \tau\right] \frac{\theta_{t_{1}, \tau}(t)}{\tau} \hat{\sigma}_{z} \hat{z}, \\
& =\frac{\hat{p}_{z}^{2}}{2 m}-\varepsilon g(t) \hat{\sigma}_{z} \hat{z},
\end{aligned}
$$

where $\hat{\sigma}_{z}$ is one of the Pauli matrices, and $\varepsilon=\mu_{B} B_{z}^{\prime}(0) \tau$. The function $\theta_{t_{1}, \tau}(t)$ is nonzero and equal to unity only inside the time interval $\left(t_{1}-\tau / 2, t_{1}+\tau / 2\right)$, i.e.,

$$
\theta_{t_{1}, \tau}(t)= \begin{cases}1, & t \in\left(t_{1}-\tau / 2, t_{1}+\tau / 2\right) \\ 0 & t \notin\left(t_{1}-\tau / 2, t_{1}+\tau / 2\right)\end{cases}
$$

and

$$
g(t)=\frac{\theta_{t_{1}, \tau}(t)}{\tau}, \quad \int_{0}^{\infty} g(t) d t=1, \quad\left(\tau \ll t_{1}\right) .
$$

We further use the simplification $g(t) \approx \delta\left(t-t_{1}\right)$ and write our model Hamiltonian as

$$
\begin{aligned}
\hat{H}(t) & =\frac{\hat{p}_{z}^{2}}{2 m}-\varepsilon \delta\left(t-t_{1}\right) \hat{\sigma}_{z} \hat{z} \\
& =\hat{H}_{0}+\hat{V}(t) \\
\hat{H}_{0} & =\hat{K}_{z} \text { is the kinetic energy operator for the } z \text { variable. }
\end{aligned}
$$

In the nomenclature introduced in the Introduction, $\hat{\sigma}_{z}$ is the observable for the system proper and $\hat{z}, \hat{p}_{z}$ are the probe canonical variables. From now on we adopt the nomen- 
clature that we measure (perhaps a better word could be "premeasure") the observable $\hat{\sigma}_{z}$, by detecting, with a suitable instrument, either $\hat{z}$ or $\hat{p}_{z}$.

We shall solve the Schrödinger equation

$$
i \hbar \frac{\partial|\psi(t)\rangle}{\partial t}=\hat{H}(t)|\psi(t)\rangle
$$

with the initial condition

$$
|\psi(0)\rangle=\left|\psi_{\text {spin }}^{(0)}\right\rangle\left|\chi^{(0)}\right\rangle
$$

Here, $\left|\psi_{\text {spin }}^{(0)}\right\rangle$ is the initial state of the system proper and $\left|\chi^{(0)}\right\rangle$ the initial state of the probe.

It will be advantageous to use the interaction picture (for a textbook presentation, see, e.g., Ref. [16]), in which we have the following relations

$$
\begin{aligned}
|\psi(t)\rangle_{I} & =\hat{U}_{0}^{\dagger}(t)|\psi(t)\rangle \\
& =\hat{U}_{0}^{\dagger}(t) \hat{U}(t)|\psi(0)\rangle \\
& \equiv \hat{U}_{I}(t)|\psi(0)\rangle \\
\hat{U}_{I}(t) & =\hat{U}_{0}^{\dagger}(t) \hat{U}(t) \\
i \hbar \frac{d \hat{U}_{I}(t)}{d t} & =\hat{V}_{I}(t) \hat{U}_{I}(t) ; \quad \hat{U}_{I}(0)=\hat{I} .
\end{aligned}
$$

Here, $\hat{U}_{0}(t), \hat{U}(t)$, are the evolution operators in the Schrödinger picture associated with $H_{0}$ and $\hat{H}$, respectively, and $\hat{U}_{I}(t)$ the evolution operator in the interaction picture; $\hat{V}_{I}(t)$ is the interaction in the interaction picture, i.e.,

$$
\begin{aligned}
\hat{V}_{I}(t) & =\mathrm{e}^{\frac{i}{\hbar} \hat{H}_{0} t} \hat{V}(t) \mathrm{e}^{-\frac{i}{\hbar} \hat{H}_{0} t} \\
& =-\varepsilon \delta\left(t-t_{1}\right) \mathrm{e}^{\frac{i}{\hbar} \hat{H}_{0} t_{1}} \hat{\sigma}_{z} \hat{z} \mathrm{e}^{-\frac{i}{\hbar} \hat{H}_{0} t_{1}} \\
& \equiv-\varepsilon \delta\left(t-t_{1}\right) \hat{W} .
\end{aligned}
$$

The solution for $\hat{U}_{I}(t)$ is

$$
\begin{aligned}
\hat{U}_{I}(t) & =\mathrm{e}^{\frac{i \varepsilon}{\hbar} \int_{0}^{t} \delta\left(t^{\prime}-t_{1}\right) d t^{\prime} \cdot \hat{W}} \\
\hat{U}_{I, f} & =\mathrm{e}^{\frac{i}{\hbar} \hat{H}_{0} t_{1}} \mathrm{e}^{\frac{i \varepsilon}{\hbar} \hat{\sigma}_{z} \hat{z}} \mathrm{e}^{-\frac{i}{\hbar} \hat{H}_{0} t_{1}} .
\end{aligned}
$$

In the last line we have the "final" evolution operator in the interaction picture, i.e., after the interaction has ceased to act. From Eq. (7d) we find the final evolution operator in the Schrödinger picture as

$$
U_{f} \equiv U\left(t>t_{1}\right)=e^{-\frac{i}{\hbar} \hat{K}_{z}\left(t-t_{1}\right)} e^{\frac{i}{\hbar} \varepsilon \hat{\sigma}_{z} \hat{z}} e^{-\frac{i}{\hbar} \hat{K}_{z} t_{1}}
$$

whose physical interpretation is very clear: we first have free evolution from $t=0$ to $t_{1}$; the interaction acts at $t=t_{1}$, and we have free evolution again thereafter. 
The final state vector, i.e., for $t>t_{1}$, is then

$$
|\Psi(t)\rangle_{f}=\sum_{\sigma= \pm 1} \mathbb{P}_{\sigma}\left|\psi_{\text {spin }}^{(0)}\right\rangle e^{-\frac{i}{\hbar} \hat{K}_{z}\left(t-t_{1}\right)} e^{\frac{i}{\hbar} \varepsilon \sigma \hat{z}}\left|\chi\left(t_{1}\right)\right\rangle
$$

We have introduced the projector $\mathbb{P}_{\sigma}$ onto the state with eigenvalue $\sigma=+1,-1$ of $\hat{\sigma}_{z}$. We observe that the component $\mathbb{P}_{\sigma}\left|\psi_{\text {spin }}^{(0)}\right\rangle$ of the original spin state gets entangled with the probe state $e^{\frac{i}{\hbar} \varepsilon \sigma \hat{z}}\left|\chi\left(t_{1}\right)\right\rangle$ (we denote by $\left|\chi\left(t_{1}\right)\right\rangle$ the probe state which has evolved freely from $t=0$ to $\left.t=t_{1}^{-}\right)$which, in the $z$-representation, is

$$
e^{\frac{i}{\hbar} \varepsilon \sigma z} \chi\left(z, t_{1}\right)
$$

meaning that it got a boost $p_{\sigma}=\varepsilon \sigma$ in the $z$ direction. This is precisely the essential physical effect occurring in the Stern-Gerlach experiment.

\subsection{The probability of detecting a probe position $z$ as a function of time}

At $t=0$, the probability density $p(z, t)$ of a probe position $z$ is just $\left|\chi^{(0)}(z)\right|^{2}$, where $\chi^{(0)}(z)$ is the original $z$-wave function. As time goes on, the wave packet spreads in time until just before the interaction occurs, i.e., until $t=t_{1}^{-}$(see the schematic illustration in Fig. 3). After the interaction has taken place at $t=t_{1}$, i.e, for $t>t_{1}$, that probability is

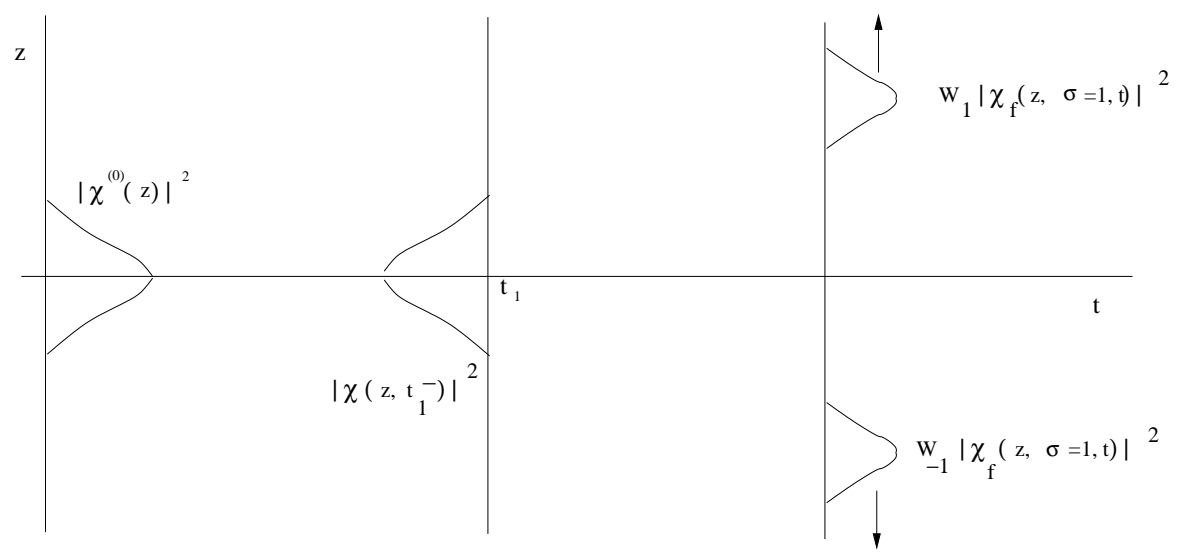

FIGURE 3. The probability $p(z, t)$ of a probe position $z$ as a function of time in the Stern-Gerlach experiment, as explained in the text.

given by

$$
\begin{aligned}
p_{f}(z, t) & ={ }_{f}\left\langle\Psi\left|\mathbb{P}_{z}\right| \Psi\right\rangle_{f} \\
& =\sum_{\sigma= \pm 1}\left\langle\psi_{\text {spin }}^{(0)}\left|\mathbb{P}_{\sigma}\right| \psi_{\text {spin }}^{(0)}\right\rangle\left|\left\langle z\left|e^{-\frac{i}{\hbar} \hat{K}_{z}\left(t-t_{1}\right)} e^{\frac{i}{\hbar} \varepsilon \sigma \hat{z}}\right| \chi\left(t_{1}\right)\right\rangle\right|^{2} \\
& =\sum_{\sigma= \pm 1} W_{\sigma}^{\left(\hat{\sigma}_{z}\right)}\left|\chi_{f}(z, \sigma ; t)\right|^{2}
\end{aligned}
$$


where $W_{\sigma}^{\left(\hat{\sigma}_{z}\right)}=\left\langle\psi_{\text {spin }}^{(0)}\left|\mathbb{P}_{\sigma}\right| \psi_{\text {spin }}^{(0)}\right\rangle$ is the Born probability for the value $\sigma$ of the spin projection in the original system state. We have also defined the $\sigma$-dependent probe wave function for $t>t_{1}$

$$
\begin{aligned}
\chi_{f}(z, \sigma ; t) & =\left\langle z\left|e^{-\frac{i}{\hbar} \hat{K}_{z}\left(t-t_{1}\right)} e^{\frac{i}{\hbar} \varepsilon \sigma \hat{z}}\right| \chi\left(t_{1}\right)\right\rangle \\
& \equiv \int U_{0}\left(z, z^{\prime} ; t-t_{1}\right) e^{\frac{i}{\hbar} \varepsilon \sigma z^{\prime}} \chi\left(z^{\prime}, t_{1}\right) d z^{\prime}
\end{aligned}
$$

which consists of the probe wave function which evolves freely up to $t=t_{1}$, it gets multiplied by the plane wave $e^{\frac{i}{\hbar} \varepsilon \sigma z^{\prime}}$ at $t=t_{1}$ (the boost referred to above), and evolves freely thereafter, through the free evolution operator indicated as $U_{0}$. Thus, because of the interaction occurring at $t=t_{1}$, the $\sigma=1$ component of this state receives a positive boost in the $z$ direction, and the $\sigma=-1$ component receives a negative boost, so that after $t=t_{1}$ they travel in opposite directions. From Eq. (12c), the $\sigma=1$ component

occurs with a weight $W_{1}^{\left(\hat{\sigma}_{z}\right)}$, and the $\sigma=-1$ component with a weight $W_{-1}^{\left(\hat{\sigma}_{z}\right)}$, as is also indicated in Fig. 3.

\subsubsection{Conditions generally required [4, 17] for the measurement of the observable of a system, when detecting a property of the probe}

What are called $\hat{A}_{s}$ and $\hat{A}_{\text {probe }}$ in Refs. [4, 17], are translated as

$$
\begin{aligned}
\hat{A}_{s} & =\hat{\sigma}_{z} \\
\hat{A}_{\text {probe }} & =\hat{z},
\end{aligned}
$$

respectively, in our notation for the present Stern-Gerlach problem. The requirements established in these references are:

i)

$$
\hat{V}=f\left(\hat{A}_{s}\right)
$$

Here, indeed: $\hat{V}=-\varepsilon \delta\left(t-t_{1}\right) \hat{\sigma}_{z} \hat{z}$

ii)

$$
\left[\hat{V}, \hat{A}_{\text {probe }}\right] \neq 0,
$$

so that $\left\langle\hat{A}_{\text {probe }}\right\rangle$ changes, enabling one to get information on $\hat{A}_{S}$ by detecting $\hat{A}_{\text {probe }}$. Although here $[\hat{V}, \hat{z}]=0$, we have $\left[\hat{K}_{z}, \hat{z}\right] \neq 0$; the kinetic energy $\hat{K}_{z}$ causes a displacement of the two wave packets, as illustrated in Fig. 3: if we wait long enough, the 2 packets separate, and we can measure $\sigma= \pm 1$.

In order to have a a QM non-demolition measurement, these references also establish the additional conditions:

iii)

$$
\begin{aligned}
{\left[\hat{V}, \hat{A}_{s}\right] } & =0 ; \quad \text { here, indeed, }\left[\hat{V}, \hat{\sigma}_{z}\right]=0 \\
{\left[\hat{H}_{s}, \hat{A}_{s}\right] } & =0 ; \quad \text { here, } H_{s}=0, \text { so that }\left[\hat{H}_{s}, \hat{\sigma}_{z}\right]=0
\end{aligned}
$$


which, taken together, give

$$
\left[\hat{H}, \hat{A}_{s}\right]=0 ; \text { here, indeed, }\left[\hat{H}, \hat{\sigma}_{z}\right]=0,
$$

with the consequence that starting with $\left|\psi_{\text {spin }}^{(0)}\right\rangle=|\sigma=1\rangle$, say, the state will not get a component $|\sigma=-1\rangle$.

\subsection{The probability of detecting a probe momentum $p_{z}$ as a function of time}

At $t=0$, the probability density $p\left(p_{z}, t\right)$ of a probe momentum $p_{z}$ is $\left|\tilde{\chi}^{(0)}\left(p_{z}\right)\right|^{2}$, where $\tilde{\chi}^{(0)}\left(p_{z}\right)$, the original wave function in the momentum representation, is the Fourier transform of the original wave function in $z$-space. As time goes on, the wave packet conserves its shape until just before the interaction occurs, i.e., until $t=t_{1}^{-}$(see the schematic illustration in Fig. 4).

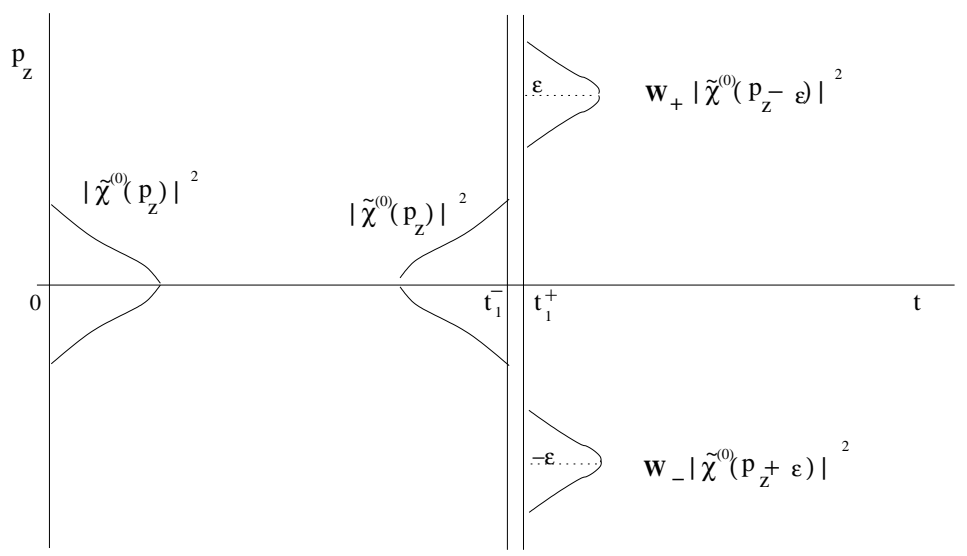

FIGURE 4. The probability $p(z, t)$ of a probe momentum $p_{z}$ as a function of time in the Stern-Gerlach experiment, as explained in the text. by

After the interaction has taken place at $t=t_{1}$, i.e, for $t>t_{1}$, that probability is given

$$
\begin{aligned}
p_{f}\left(p_{z}, t\right) & ={ }_{f}\left\langle\Psi\left|\mathbb{P}_{p_{z}}\right| \Psi\right\rangle_{f} \\
& =\sum_{\sigma= \pm 1}\left\langle\psi_{\text {spin }}^{(0)}\left|\mathbb{P}_{\sigma}\right| \psi_{\text {spin }}^{(0)}\right\rangle\left|\left\langle p_{z}\left|e^{-\frac{i}{\hbar} \hat{K}_{z}\left(t-t_{1}\right)} e^{\frac{i}{\hbar} \varepsilon \sigma \hat{z}}\right| \chi\left(t_{1}\right)\right\rangle\right|^{2} \\
& =\sum_{\sigma= \pm 1} W_{\sigma}^{\left(\hat{\sigma}_{z}\right)}\left|\tilde{\chi}^{(0)}\left(p_{z}-\varepsilon \sigma\right)\right|^{2} .
\end{aligned}
$$

Just as in Eq. (12), $W_{\sigma}^{\left(\hat{\sigma}_{z}\right)}$ is the Born probability for the value $\sigma$ of the spin projection in the original system state; $\tilde{\chi}^{(0)}\left(p_{z}-\varepsilon \sigma\right)$ is the original wave function in momentum space, evaluated at the displaced value $p_{z}-\varepsilon \sigma$. Thus, at $t=t_{1}^{+}$the $p_{z}$ probability density splits into the two pieces indicated in Fig. 4, corresponding to the two values of $\sigma$, with weights $W_{+1}^{\left(\hat{\sigma}_{z}\right)}$ and $W_{-1}^{\left(\hat{\sigma}_{z}\right)}$, respectively, and remains unaltered thereafter. 


\subsubsection{Conditions generally required $[4,17]$ for the measurement of the} observable of a system, when detecting a property of the probe

In this case, the $\hat{A}_{s}$ and $\hat{A}_{\text {probe }}$ of Refs. $[4,17]$ are

$$
\begin{aligned}
\hat{A}_{s} & =\hat{\sigma}_{z} \\
\hat{A}_{\text {probe }} & =\hat{p}_{z},
\end{aligned}
$$

respectively, in our present notation. We recall that these references require:

i)

$$
\hat{V}=f\left(\hat{A}_{s}\right)
$$

indeed, this is the case, because $\hat{V}=-\varepsilon \delta\left(t-t_{1}\right) \hat{\sigma}_{z} \hat{z}$.

ii)

$$
\left[\hat{V}, \hat{A}_{\text {probe }}\right] \neq 0 \text {. }
$$

Here, indeed, $\left[\hat{V}, \hat{p}_{z}\right] \propto\left[\hat{z}, \hat{p}_{z}\right] \neq 0$.

For a QM non-demolition measurement, these references require:

iii)

$$
\begin{aligned}
{\left[\hat{V}, \hat{A}_{s}\right] } & =0 ; \text { here, indeed }:\left[\hat{V}, \hat{\sigma}_{z}\right]=0 \\
{\left[\hat{H}_{s}, \hat{A}_{s}\right] } & =0 ; \text { here, } H_{s}=0, \text { so that }\left[\hat{H}_{s}, \hat{\sigma}_{z}\right]=0,
\end{aligned}
$$

so that

$$
\left[\hat{H}, \hat{A}_{s}\right]=0 ; \text { here, indeed }:\left[\hat{H}, \hat{\sigma}_{z}\right]=0 \text {. }
$$

Just as in the previous case, the consequence is that starting with $\left|\psi_{\text {spin }}^{(0)}\right\rangle=|\sigma=1\rangle$, say, the state will not get a component $|\sigma=-1\rangle$.

\section{SINGLE MEASUREMENTS IN QUANTUM MECHANICS}

We first consider the measurement of an observable $\hat{A}$ using one probe and detecting a property of it. We are using the nomenclature introduced right after Eqs. (5).

For $\hat{A}$ we write the spectral representation

$$
\hat{A}=\sum_{n} a_{n} \mathbb{P}_{a_{n}}
$$

where the eigenvalues $a_{n}$ are allowed to be degenerate and $\mathbb{P}_{a_{n}}$ are the eigenprojectors.

We assume the system to be coupled to a probe, considered, for simplicity, to be onedimensional, whose position and momentum are represented by the Hermitean operators $\hat{Q}$ and $\hat{P}$. The system-probe interaction is taken to be [1]

$$
\hat{V}(t)=\varepsilon g(t) \hat{A} \hat{P}, \quad t_{1}>0,
$$

with an arbitrary interaction strength [18] $\varepsilon$. 
This interaction could be translated to the one for the Stern-Gerlach experiment discussed in Sec. 2.2 using the correspondence $\hat{A} \Rightarrow \hat{\sigma}_{z}, \hat{P} \Rightarrow \hat{z}, \hat{Q} \Rightarrow-\hat{p}_{z}$, as illustrated in Fig. 5 below. The delta function interaction of the previous section was generalized to $g(t)$ (see Eq. (4)), a narrow function with finite support, centered at $t=t_{1}$, so that

$$
\begin{aligned}
\int_{0}^{t} g\left(t^{\prime}\right) d t^{\prime} & \equiv G(t), \\
G(0) & =0, \quad G(\infty)=1 .
\end{aligned}
$$

We disregard the intrinsic evolution of the system and the probe, and assume that $\hat{V}(t)$ of Eq. (24) represents the full Hamiltonian, i.e.,

$$
\hat{H}(t)=\varepsilon g(t) \hat{A} \hat{P}, \quad t_{1}>0 .
$$

The evolution operator is then given by

$$
\hat{U}(t)=\mathrm{e}^{-\frac{i}{\hbar} \int_{0}^{t} \hat{H}\left(t^{\prime}\right) d t^{\prime}}=\mathrm{e}^{-\frac{i}{\hbar} \varepsilon G(t) \hat{A} \hat{P}} .
$$

If the density operator of the system plus the probe at $t=0$ is the direct product $\rho^{(0)}=\rho_{s}^{(0)} \otimes \rho_{\pi}^{(0)}$ ( $\pi$ stands for "probe"), after the interaction has ceased to act, i.e., for $t \gg t_{1}$, it is given by

$$
\rho_{f}^{(\hat{A})}=\sum_{n n^{\prime}} \mathbb{P}_{a_{n}} \rho_{s}^{(0)} \mathbb{P}_{a_{n^{\prime}}}\left(e^{-\frac{i}{\hbar} \varepsilon a_{n} \hat{P}} \rho_{\pi}^{(0)} e^{\frac{i}{\hbar} \varepsilon a_{n^{\prime}} \hat{P}}\right)
$$

From this expression we notice that, because of the interaction, the system and the probe are now correlated. Also notice the presence of the displacement operator $\exp \left(-(i / \hbar) \varepsilon a_{n} \hat{P}\right)$ in this last equation.

Now the idea is that at time $t>t_{1}$, i.e., after the system-probe interaction is over, we detect the probe position $\hat{Q}$ to obtain information on the system proper. This we study in what follows.

\subsection{The $\hat{Q}$ probability density after the interaction}

According to Born's rule, the $Q$ probability density for $t>t_{1}$ is given by

$$
p_{f}^{(\hat{A})}(Q)=\operatorname{Tr}\left(\rho_{f}^{(\hat{A})} \mathbb{P}_{Q}\right)=\sum_{n} W_{a_{n}}^{(\hat{A})} p_{0}\left(Q-\varepsilon a_{n}\right),
$$

where

$$
W_{a_{n}}^{(\hat{A})}=\operatorname{Tr}\left(\rho_{s}^{(0)} \mathbb{P}_{a_{n}}\right)
$$

is the Born probability for the result $a_{n}$ in the original system state, and

$$
p_{0}\left(Q-\varepsilon a_{n}\right)=\left\langle Q-\varepsilon a_{n}\left|\rho_{\pi}^{(0)}\right| Q-\varepsilon a_{n}\right\rangle
$$


is the original $Q$ probability density $p_{0}(Q)$ (which has a width $=\sigma_{Q}$ ), but displaced by $\varepsilon a_{n}$.

In this problem we may take the point of view that knowing the system state $\rho_{S}^{(0)}$ before the process, and thus $W_{a_{n}}^{(\hat{A})}$, we can predict the detectable quantity $p_{f}^{(\hat{A})}(Q)$. We may also adopt the more interesting viewpoint that, detecting $p_{f}^{(\hat{A})}(Q)$, we can retrieve information on the system state. We examine this latter attitude below.

Before doing that, we illustrate in Fig. 5 the result (29) for the case of the SternGerlach experiment studied in the last section, by means of the translation given right before Eq. (25).

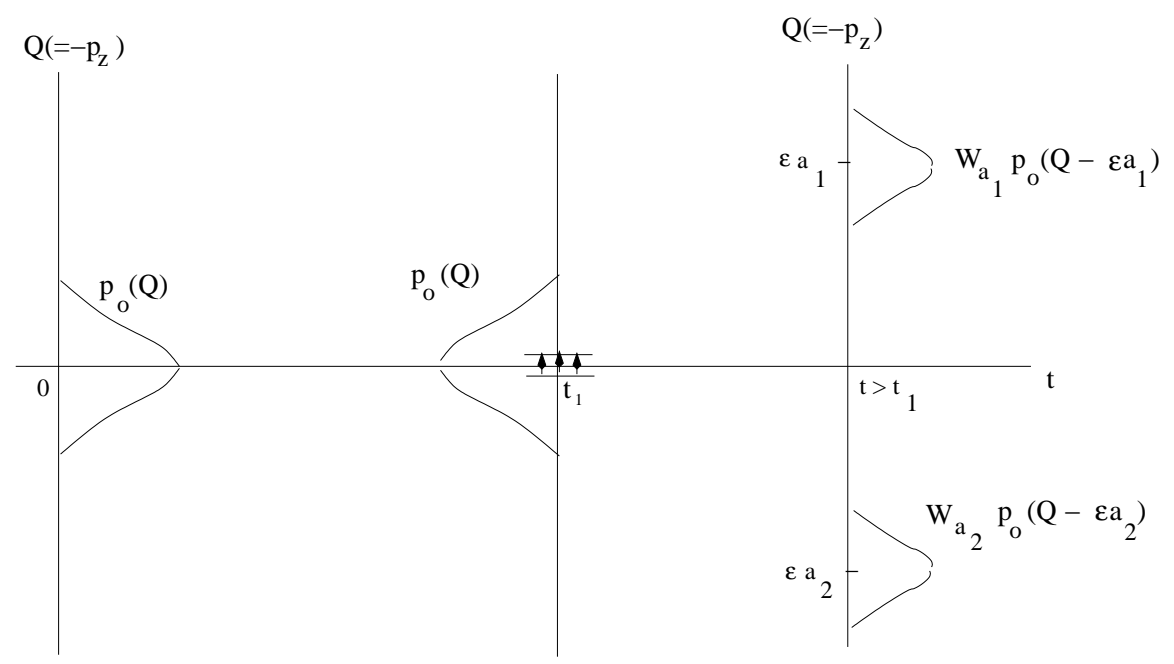

FIGURE 5. The probability density $p_{f}^{(\hat{A})}(Q)$ of the probe degree of freedom $Q=-p_{z}$ as a function of time in the Stern-Gerlach experiment, as explained in the text. This is basically Fig. 4, with the translation of variables $\hat{A} \Rightarrow \hat{\sigma}_{z}, \hat{P} \Rightarrow \hat{z}, \hat{Q} \Rightarrow-\hat{p}_{z}$.

An illustrative example of a more general case is presented in Fig. 6; for the values of the parameters indicated in the figure, Fig. 6a corresponds to the case of "strong coupling", while Fig. $6 \mathrm{~b}$ to that of "weak coupling".

In the above scheme, what we detect is the probe-position probability $p_{f}^{(\hat{A})}(Q)$. As it can be seen from Fig. 6, it is only in the idealized limit of very strong coupling, $\varepsilon / \sigma_{Q} \gg 1$, that $p_{f}^{(\hat{A})}(Q)$ "mirrors" the eigenvalues $a_{n}$ of the observable which we want to have information about. In this limit, $p_{f}^{(\hat{A})}(Q)$ integrated around $a_{n}$ gives Born's probability $W_{a_{n}}^{(\hat{A})}$ of $a_{n}$. In this high-resolution limit this is thus the information we can retrieve about the system proper, by detecting $Q$. We shall study below what information can be extracted from experiments with arbitrary resolution. Surprisingly, we shall find cases where it is advantageous to use low resolution (see Sec. 5, last paragraph)! 

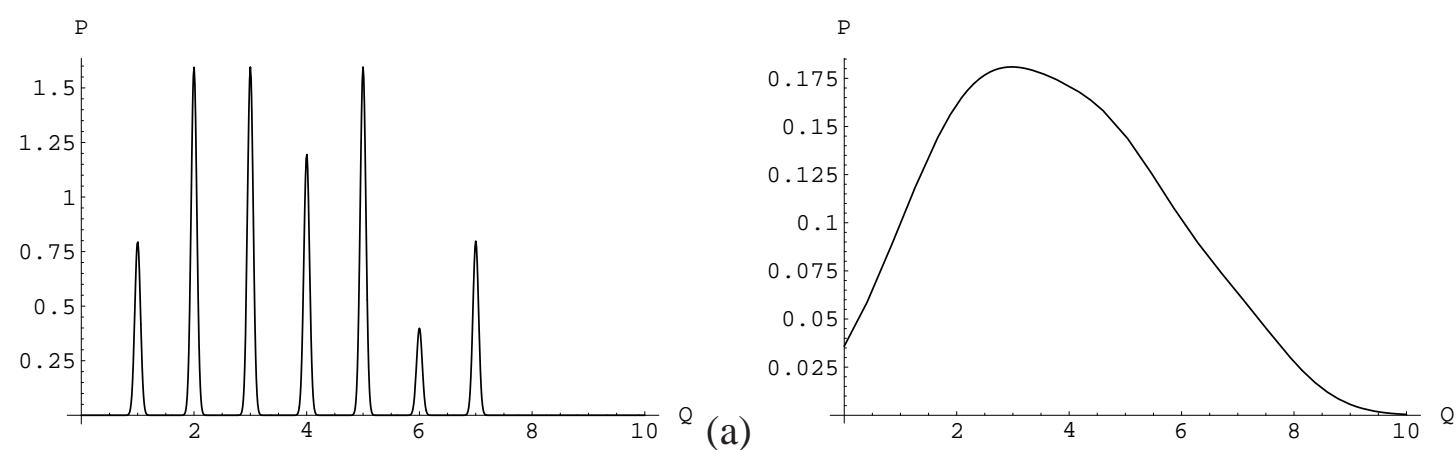

FIGURE 6. Illustrative example of the probability density of the pointer position $Q$ of Eq. (29). We have assumed seven eigenvalues $a_{n}$ for the system observable $\hat{A}$, with Born probabilities [Eq. (30)] given by: $0.1,0.2,0.2,0.15,0.2,0.05$ and 0.1 . We chose the particular values: (a) $\varepsilon=1$ for the interaction strength and $\sigma_{Q}=0.05$ for the width of the probe state prior to the measurement; this is a case of "strong coupling"; (b) $\varepsilon=1$ for the interaction strength and $\sigma_{Q}=1$ for the width of the probe state prior to the measurement; this is a case of "weak coupling".

\subsection{The average of $Q$ after the interaction}

From Eqs. (29) and (30) one finds [19] that the average of the probe position $\hat{Q}$ in units of $\varepsilon$, after the interaction is over, is given by

$$
\frac{1}{\varepsilon}\langle\hat{Q}\rangle_{f}^{(\hat{A})}=\sum_{n} a_{n} W_{a_{n}}^{(\hat{A})}=\sum_{n} a_{n} \operatorname{Tr}\left(\rho_{s}^{(0)} \mathbb{P}_{a_{n}}\right)=\operatorname{Tr}\left(\rho_{s}^{(0)} \hat{A}\right)=\langle\hat{A}\rangle_{0}, \quad \forall \varepsilon
$$

We have assumed the original $Q$ distribution to be centered at $Q=0$. As a result, detecting the average probe position $\langle\hat{Q}\rangle_{f}^{(\hat{A})}$ after the interaction is over allows extracting the Born average $\langle\hat{A}\rangle_{0}$ of the observable $\hat{A}$ in the original state of the system. It is remarkable that this result is valid for arbitrary coupling strength $\varepsilon$. For example, in the two situations illustrated in Fig. 6 we would obtain the same result for $\langle\hat{Q}\rangle_{f}^{(\hat{A})} / \varepsilon$.

Similar results can be found for higher-order moments. E. g., for the second moment of $\hat{Q}$ one finds

$$
\frac{1}{\varepsilon^{2}}\left[\left\langle\hat{Q}^{2}\right\rangle_{f}^{(\hat{A})}-\sigma_{Q}^{2}\right]=\operatorname{Tr}\left(\rho_{s}^{(0)} \hat{A}^{2}\right)=\left\langle\hat{A}^{2}\right\rangle_{0}, \quad \forall \varepsilon
$$

implying that detecting $\left\langle\hat{Q}^{2}\right\rangle_{f}^{(\hat{A})}$ and knowing $\sigma_{Q}^{2}$ allows extracting the second moment of the observable $\hat{A}$ in the original state of the system, i.e., $\left\langle\hat{A}^{2}\right\rangle_{0}$.

More in general, if we detect the final $Q$ probability density (29), i.e.,

$$
p_{f}^{(\hat{A})}(Q)=\sum_{n}\left(\rho_{s}^{(0)}\right)_{n n} p_{0}\left(Q-\varepsilon a_{n}\right)
$$

we obtain information on the diagonal elements $\left(\rho_{s}^{(0)}\right)_{n n}$ of the original density operator, but not on the off-diagonal ones. Alternatively, we can write this result in terms of the 
characteristic function

$$
\tilde{p}_{f}^{(\hat{A})}(k)=\left[\sum_{n}\left(\rho_{s}^{(0)}\right)_{n n} \mathrm{e}^{i k \varepsilon a_{n}}\right] \tilde{p}_{0}(k)=\left\langle\mathrm{e}^{i k \varepsilon \hat{A}}\right\rangle_{0} \tilde{p}_{0}(k),
$$

implying that if we detect $p_{f}^{(\hat{A})}(Q)$ and infer $\tilde{p}_{f}^{(\hat{A})}(k)$, we can extract $\left\langle\mathrm{e}^{i k \varepsilon \hat{A}}\right\rangle_{0}$. Results (32) and (33) are particular cases of Eq. (35).

In Sec. 5 we shall find a procedure to extract all of the matrix elements of the original density operator, using the notion of successive measurements.

\subsection{Measuring projectors}

A particular case of great interest is the measurement of a projector, like $\mathbb{P}_{a_{v}}$, so that the Hamiltonian of Eq. (26) becomes

$$
\hat{H}(t)=\varepsilon g(t) \hat{\mathbb{P}}_{a_{v}} \hat{P}, \quad t_{1}>0 .
$$

We designate the eigenvalues of $\hat{\mathbb{P}}_{a_{v}}$ by $\tau=1,0$, and its eigenprojectors by $\left(\hat{\mathbb{P}}_{a_{v}}\right) \tau$. Then

$$
\begin{aligned}
& \left(\hat{\mathbb{P}}_{a_{v}}\right)_{1}=\hat{\mathbb{P}}_{a_{v}} ; \quad \hat{\mathbb{P}}_{a_{v}}\left(\hat{\mathbb{P}}_{a_{v}}\right)_{1}=1 \cdot\left(\hat{\mathbb{P}}_{a_{v}}\right)_{1} \\
& \left(\hat{\mathbb{P}}_{a_{v}}\right)_{0}=I-\hat{\mathbb{P}}_{a_{v}} ; \quad \hat{\mathbb{P}}_{a_{v}}\left(\hat{\mathbb{P}}_{a_{v}}\right)_{0}=0 \cdot\left(\hat{\mathbb{P}}_{a_{v}}\right)_{0} .
\end{aligned}
$$

For these eigenvalues and eigenprojectors, the probe-position probability density of Eq. (29) gives

$$
p_{f}^{\left(\hat{\mathbb{P}}_{a_{v}}\right)}(Q)=\operatorname{Tr}\left[\rho_{s}^{(0)}\left(\hat{\mathbb{P}}_{a_{v}}\right)_{0}\right] p_{0}(Q)+\operatorname{Tr}\left[\rho_{s}^{(0)}\left(\hat{\mathbb{P}}_{a_{v}}\right)_{1}\right] p_{0}(Q-\varepsilon) .
$$

This result is illustrated in Fig. 7 for the strong-coupling case, in which $p_{f}^{\left(\hat{\mathbb{P}}_{a_{v}}\right)}(Q)$ consists of two peaks centered at $Q / \varepsilon=0$ and $Q / \varepsilon=1$. From Eq. (38), or from Eq.

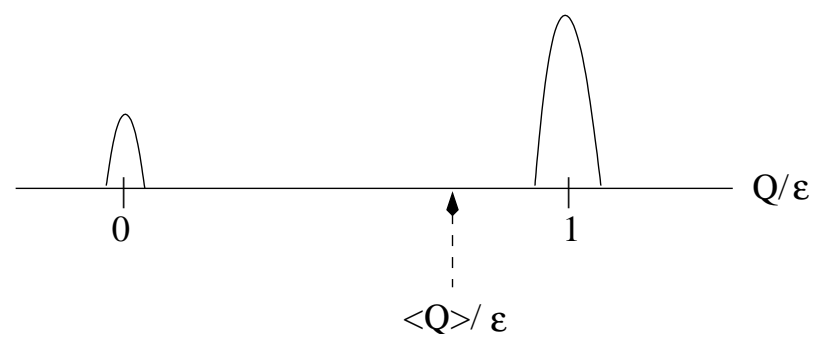

FIGURE 7. Illustrative example (qualitative) of the probability density of the probe position $Q$ of Eq. (38) for the strong-coupling case. The result consists of two peaks, centered at $Q / \varepsilon=0$ and $Q / \varepsilon=1$. Also shown is the average of the probe position which, in units of $\varepsilon$, is independent of $\varepsilon$ and lies between 0 and 1.

(32), we find

$$
\frac{1}{\varepsilon}\langle\hat{Q}\rangle_{f}^{\left(\hat{\mathbb{P}}_{a_{v}}\right)}=\sum_{\tau=0}^{1} \tau \operatorname{Tr}\left[\rho_{s}^{(0)}\left(\hat{\mathbb{P}}_{a_{v}}\right)_{\tau}\right]=\left\{\begin{array}{l}
\operatorname{Tr}\left(\rho_{s}^{(0)} \hat{\mathbb{P}}_{a_{v}}\right)=W_{a_{v}}^{(\hat{A})} \\
\operatorname{Tr}\left[\rho_{s}^{(0)}\left(\hat{\mathbb{P}}_{a_{v}}\right)_{\tau=1}\right]=W_{\tau=1}^{\left(\hat{\mathbb{P}}_{a_{v}}\right)}
\end{array}\right.
$$


a result independent of $\varepsilon$. Thus the final average probe position, in units of $\varepsilon$, gives directly the probability $W_{a_{v}}^{(\hat{A})}$ of $a_{v}$ of the observable $\hat{A}$ in the original state (see the first row of Eq. (39), where we used $\left.\sum_{\tau} \tau\left(\hat{\mathbb{P}}_{a_{v}}\right)_{\tau}=\hat{\mathbb{P}}_{a_{v}}\right)$; this is illustrated by the arrow in Fig.

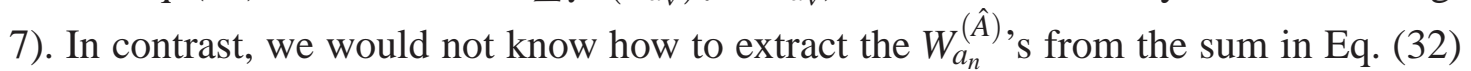
if $\hat{A}$ is not a projector. We can also say that inferring the probability of the eigenvalue $\tau=1$ (second row in (39)), i.e., the probability of "yes" of the observable $\hat{\mathbb{P}}_{a_{v}}$, from a detection of $\langle\hat{Q}\rangle_{f}^{\left(\hat{\mathbb{P}}_{a_{v}}\right)} / \varepsilon$ (LHS of (39)), is equivalent to retrieving the diagonal elements $\left\langle a_{v}\left|\rho_{s}^{(0)}\right| a_{v}\right\rangle$ of the original density operator of the system (first row in (39)). As we shall see in Sec. 5, the extension of this last idea to successive measurements will allow retrieving the full density operator.

\subsection{The reduced density operator of the system proper after the interaction}

We compute the reduced density operator of the system proper after the interaction with the probe is over, by tracing $\rho_{f}^{(\hat{A})}$ of Eq. (28) over the probe, with the result

$$
\begin{aligned}
\rho_{s, f}^{(\hat{A})} & =\sum_{n n^{\prime}}\left(\mathbb{P}_{a_{n}} \rho_{s}^{(0)} \mathbb{P}_{a_{n^{\prime}}}\right) \operatorname{Tr}_{\pi}\left[e^{-\frac{i}{\hbar} \varepsilon a_{n} \hat{P}} \rho_{\pi}^{(0)} e^{\frac{i}{\hbar} \varepsilon a_{n^{\prime}} \hat{P}}\right] \\
& =\sum_{n, n^{\prime}} g\left(\varepsilon\left(a_{n}-a_{n^{\prime}}\right)\right) \mathbb{P}_{a_{n}} \rho_{s}^{(0)} \mathbb{P}_{a_{n^{\prime}}}
\end{aligned}
$$

where we have defined the characteristic function of the probe momentum distribution as

$$
g(\beta)=\left\langle\mathrm{e}^{-\frac{i}{\hbar} \beta \hat{P}}\right\rangle_{\pi}^{(0)}=\operatorname{Tr}\left[\rho_{\pi}^{(0)} e^{-\frac{i}{\hbar} \beta \hat{P}}\right] ; \quad \beta=\varepsilon\left(a_{n}-a_{n^{\prime}}\right)
$$

As an example, for the particular case of a Gaussian state for the probe we have

$$
\begin{aligned}
\chi(Q) & =\frac{e^{-\frac{Q^{2}}{4 \sigma_{Q}^{2}}}}{\left(2 \pi \sigma_{Q}^{2}\right)^{1 / 4}} \\
g\left(\varepsilon\left(a_{n}-a_{n^{\prime}}\right)\right) & =\int \chi^{*}\left(Q-\varepsilon a_{n^{\prime}}\right) \chi\left(Q-\varepsilon a_{n}\right) d Q \\
& =e^{-\frac{\varepsilon^{2}}{8 \sigma_{Q}^{2}}\left(a_{n}-a_{n^{\prime}}\right)^{2}}=e^{-\frac{1}{2}\left(\frac{\varepsilon \sigma_{P}}{\hbar}\right)^{2}\left(a_{n}-a_{n^{\prime}}\right)^{2}} .
\end{aligned}
$$

The result (40) is valid for an arbitrary state of the probe and an artbitrary coupling strength $\varepsilon / \sigma_{Q}$. In the strong-coupling limit $\varepsilon / \sigma_{Q} \rightarrow \infty, \rho_{s, f}^{(\hat{A})}$ reduces to

$$
\rho_{s, f}^{(\hat{A})}=\sum_{n} \mathbb{P}_{a_{n}} \rho_{s}^{(0)} \mathbb{P}_{a_{n}}
$$


This is called the von Neumann-Lüders rule, originally postulated by Lüders [8], and then given a dynamical derivation in Ref. [20] using vNM.

We have thus reproduced the result of a non-selective projective measurement [21] of the observable $\hat{A}$, as a limiting case of our general formalism.

Notice that $\rho_{s, f}^{(\hat{A})}$ and $\rho_{s}^{(0)}$ are not connected by a unitary transformation: indeed, their eigenvalues have changed. For example, in the particular case in which the initial system state is the pure state $\rho_{s}^{(0)}=\left|\psi_{s}^{(0)}\right\rangle\left\langle\psi_{s}^{(0)}\right|$, the initial eigenvalues are $1,0, \cdots, 0$. On the other hand, one eigenstate of $\rho_{s, f}^{(\hat{A})}$ is $\mathbb{P}_{a_{v}}\left|\psi_{s}^{(0)}\right\rangle$, fulfilling the eigenvalue equation

$$
\rho_{s, f}^{(\hat{A})}\left(\mathbb{P}_{a_{v}}\left|\psi_{s}^{(0)}\right\rangle\right)=\left\langle\psi_{s}^{(0)}\left|\mathbb{P}_{a_{v}}\right| \psi_{s}^{(0)}\right\rangle\left(\mathbb{P}_{a_{v}}\left|\psi_{s}^{(0)}\right\rangle\right)
$$

so that the corresponding eigenvalue is $\left\langle\psi_{s}^{(0)}\left|\mathbb{P}_{a_{v}}\right| \psi_{s}^{(0)}\right\rangle$. This is not a contradiction, because it is the density operator for the whole system, i.e., the system proper plus the probe, that evolves unitarily (see Eqn. (28)), while here we are dealing with the reduced density operator, which is the full density operator traced over the probe.

We computed above the reduced density matrix for the system proper, $\rho_{s, f}^{(\hat{A})}$, after the system-probe interaction; then the probe is detected. We now wish to make a model for the probe-detector $(\pi-D)$ interaction, taking place at some time $t_{2}>t_{1}$, and investigate if the resulting reduced density matrix for the system proper is still the same as the one given above, in Eq. (40). Assume that this new interaction does not involve the system proper $s$. The final density operator after the interaction with the detector, to be called $\rho_{f}^{\text {(after inter. with detector) }}$, will contain a new evolution operator $U_{\pi D}$, that involves the probe and the detector, but not the system $s$. Tracing $\rho_{f}^{\text {(after inter. with detector) }}$ over the probe and the detector, we obtain

$$
\begin{aligned}
\rho_{s, f}^{(\text {after inter. with detector })} & =\operatorname{Tr}_{\pi D}\left(\rho_{f}^{(\text {after inter. with detector })}\right) \\
& =\sum_{n n^{\prime}}\left(\mathbb{P}_{a_{n}} \rho_{s}^{(0)} \mathbb{P}_{a_{n^{\prime}}}\right) \operatorname{Tr}_{\pi D}\left[U_{\pi D} e^{-\frac{i}{\hbar} \varepsilon a_{n} \hat{P}} \rho_{\pi}^{(0)} e^{\frac{i}{\hbar} \varepsilon a_{n^{\prime}} \hat{P}} \hat{\rho}_{D}^{(0)} U_{\pi D}^{\dagger}\right] \\
& =\sum_{n n^{\prime}} g\left(\varepsilon\left(a_{n}-a_{n^{\prime}}\right)\right) \mathbb{P}_{a_{n}} \rho_{s}^{(0)} \mathbb{P}_{a_{n^{\prime}}}
\end{aligned}
$$

giving the same answer as before, Eq. (40). We stress that this result holds when the probe-detector interaction does not involve $s$.

\section{SUCCESSIVE MEASUREMENTS IN QUANTUM MECHANICS}

We now generalize the problem of the previous section to describe the measurement of two observables in succession: $\hat{A}$, as defined in Eq. (23), at time $t_{1}$, and then

$$
\hat{B}=\sum_{m} b_{m} \mathbb{P}_{b_{m}},
$$


(the $b_{m}$ 's may also be degenerate), at some later time $t_{2}$. For this purpose, we assume that we employ two probes, which are the ones that we detect; their momentum and coordinate operators are $\hat{P}_{i}, \hat{Q}_{i}, i=1,2$. The interaction of the system with the probes defines the Hamiltonian

$$
\hat{H}(t)=\varepsilon_{1} g_{1}(t) \hat{A} \hat{P}_{1}+\varepsilon_{2} g_{2}(t) \hat{B} \hat{P}_{2} .
$$

Again, we have disregarded the intrinsic Hamiltonians of the system and of the two probes. The functions $g_{1}(t)$ and $g_{2}(t)$ are narrow non-overlapping functions, centered around $t=t_{1}$ and $t=t_{2}$, respectively (see Eqs. (25)), with $0<t_{1}<t_{2}$.

The unitary evolution operator is given by

$$
\hat{U}(t)=\mathrm{e}^{-\frac{i}{\hbar} \varepsilon_{2} G_{2}(t) \hat{B} \hat{P}_{2}} \mathrm{e}^{-\frac{i}{\hbar} \varepsilon_{1} G_{1}(t) \hat{A} \hat{P}_{1}} .
$$

If the density operator of the system plus the probes at $t=0$ is assumed to be the direct product $\rho^{(0)}=\rho_{s} \otimes \rho_{\pi_{1}} \otimes \rho_{\pi_{2}}$, for $t \gg t_{2}$, i.e., after the second interaction has ceased to act, it is given by

$$
\begin{aligned}
& \rho_{f}^{(\hat{B} \leftarrow \hat{A})}=\sum_{n n^{\prime} m m^{\prime}}\left(\mathbb{P}_{b_{m}} \mathbb{P}_{a_{n}} \rho_{s}^{(0)} \mathbb{P}_{a_{n^{\prime}}} \mathbb{P}_{b_{m^{\prime}}}\right) \\
& \quad \cdot\left(e^{-\frac{i}{\hbar} \varepsilon_{1} a_{n} \hat{P}_{1}} \rho_{\pi_{1}}^{(0)} e^{\frac{i}{\hbar} \varepsilon_{1} a_{n^{\prime}} \hat{P}_{1}}\right)\left(e^{-\frac{i}{\hbar} \varepsilon_{2} b_{m} \hat{P}_{2}} \rho_{\pi_{2}}^{(0)} e^{\frac{i}{\hbar} \varepsilon_{2} b_{m^{\prime}} \hat{P}_{2}}\right) .
\end{aligned}
$$

At $t \gg t_{2}$ we detect the two probe positions and momenta in order to obtain information about the system. Two examples are considered below.

We first detect the two probe positions $\hat{Q}_{1}$ and $\hat{Q}_{2}$. Their correlation can be calculated as

$$
\left\langle\hat{Q}_{1} \hat{Q}_{2}\right\rangle_{f}^{(\hat{B} \leftarrow \hat{A})}=\operatorname{Tr}\left[\rho_{f}^{(\hat{B} \leftarrow \hat{A})} \hat{Q}_{1} \hat{Q}_{2}\right]
$$

with the result $[7,9]$

$$
\frac{\left\langle\hat{Q}_{1} \hat{Q}_{2}\right\rangle_{f}^{(\hat{B} \leftarrow \hat{A})}}{\varepsilon_{1} \varepsilon_{2}}=\Re \sum_{n m} a_{n} b_{m} W_{b_{m} a_{n}}^{(\hat{B} \leftarrow \hat{A})}\left(\varepsilon_{1}\right)
$$

where $\mathfrak{R}$ stands for the real part. We have defined

$$
W_{b_{m} a_{n}}^{(\hat{B} \leftarrow \hat{A})}\left(\varepsilon_{1}\right)=\sum_{n^{\prime}} \lambda\left(\varepsilon_{1}\left(a_{n}-a_{n^{\prime}}\right)\right) \operatorname{Tr}\left[\rho_{s}^{(0)}\left(\mathbb{P}_{a_{n^{\prime}}} \mathbb{P}_{b_{m}} \mathbb{P}_{a_{n}}\right)\right]
$$

and

$$
\begin{aligned}
\lambda(\beta) & =g(\beta)+2 h(\beta), \\
g(\beta) & =\left\langle\mathrm{e}^{-\frac{i}{\hbar} \beta \hat{P}_{1}}\right\rangle_{\pi_{1}}^{(0)}, \\
h(\beta) & =\frac{1}{\beta}\left\langle\mathrm{e}^{-\frac{i}{2 \hbar} \beta \hat{P}_{1}} \hat{Q}_{1} \mathrm{e}^{-\frac{i}{2 \hbar} \beta \hat{P}_{1}}\right\rangle_{\pi_{1}}^{(0)} .
\end{aligned}
$$


Here, $\langle\cdots\rangle_{\pi_{1}}^{(0)}$ indicates an average over the initial state of probe 1 . Notice that $\left\langle\hat{Q}_{1} \hat{Q}_{2}\right\rangle_{f}^{(\hat{B} \leftarrow \hat{A})}$ may be a complicated function of $\varepsilon_{1}$; however, it is linear in $\varepsilon_{2}$, the strength associated with the last measurement, just as for a single measurement we found, in Eq. (32), that $\langle\hat{Q}\rangle_{f}^{(\hat{A})} \propto \varepsilon$. We also have the result (see Ref. [9] for the relevant conditions)

$$
\lambda(0)=1 .
$$

The following comments are in order at this point. Knowing the original system state $\rho_{s}^{(0)}$, the auxiliary function $\mathfrak{R} W_{b_{m} a_{n}}^{(\hat{A} \leftarrow \hat{A})}\left(\varepsilon_{1}\right)$ appearing in Eq. (51) allows predicting the detectable quantity $\left\langle\hat{Q}_{1} \hat{Q}_{2}\right\rangle_{f}^{(\hat{B} \leftarrow \hat{A})}$. It extends to two measurements the Born probability $W_{a_{n}}^{(\hat{A})}=\operatorname{Tr}\left(\rho_{s}^{(0)} \mathbb{P}_{a_{n}}\right)$ of Eq. (30) which, for single measurements, allows predicting the detectable quantity $\langle\hat{Q}\rangle_{f}^{(\hat{A})}=\varepsilon \sum_{n} a_{n} W_{a_{n}}^{(\hat{A})}$ of Eq. (32). More interestingly, detecting $\left\langle\hat{Q}_{1} \hat{Q}_{2}\right\rangle_{f}^{(\hat{B} \leftarrow \hat{A})}$, we investigate what information can we retrieve on the system state. This is the point of view that will be taken in the next section.

As the next example, we consider again the same Hamiltonian of Eq. (47) but, after the second interaction has acted, i.e., for $t \gg t_{2}$, we detect, on a second sub-ensemble, the momentum $\hat{P}_{1}$ of the first probe instead of its position, and the position $\hat{Q}_{2}$ of the second probe. The resulting correlation between $\hat{P}_{1}$ and $\hat{Q}_{2}$ is $[7,9]$

$$
\frac{1}{\varepsilon_{1} \varepsilon_{2}}\left\langle\hat{P}_{1} \hat{Q}_{2}\right\rangle^{(\hat{B} \leftarrow \hat{A})}=\frac{1}{2 \sigma_{Q_{1}}^{2}} \mathfrak{I} \sum_{n m} a_{n} b_{m} \tilde{W}_{b_{m} a_{n}}^{(\hat{B} \leftarrow \hat{A})}\left(\varepsilon_{1}\right),
$$

where

$$
\tilde{W}_{b_{m} a_{n}}^{(\hat{B} \leftarrow \hat{A})}\left(\varepsilon_{1}\right)=\sum_{n^{\prime}} \tilde{\lambda}\left(\varepsilon_{1}\left(a_{n}-a_{n^{\prime}}\right)\right) \operatorname{Tr}\left[\rho_{s}^{(0)}\left(\mathbb{P}_{a_{n^{\prime}}} \mathbb{P}_{b_{m}} \mathbb{P}_{a_{n}}\right)\right]
$$

and

$$
\begin{aligned}
\tilde{\lambda}(\beta) & =\frac{\bar{\lambda}(\beta)}{\bar{\lambda}(0)}, \\
\bar{\lambda}(\beta) & =\frac{1}{\beta} \frac{\partial g(\beta)}{\partial \beta}
\end{aligned}
$$

Just as in the first example, the auxiliary function $\mathfrak{I} \tilde{W}_{b_{m} a_{n}}^{(\hat{B} \leftarrow \hat{A})}\left(\varepsilon_{1}\right)$ allows predicting the detectable quantity $\left\langle\hat{P}_{1} \hat{Q}_{2}\right\rangle_{f}^{(\hat{B} \leftarrow \hat{A})}$. Alternatively, detecting $\left\langle\hat{P}_{1} \hat{Q}_{2}\right\rangle_{f}^{(\hat{B} \leftarrow \hat{A})}$, we shall investigate what information can we retrieve on the system state.

We illustrate in Fig. 8 the measurements of Eqs. (51) and (55) for the particular case of the Stern-Gerlach experiment studied in section 2, using the following translation of observables:

$$
\begin{array}{cc}
\hat{A} \Rightarrow \hat{\sigma}_{z} & \hat{B} \Rightarrow \hat{\sigma}_{x} \\
\hat{P}_{1} \Rightarrow \hat{z} & \hat{P}_{2} \Rightarrow \hat{x} \\
\hat{Q}_{1} \Rightarrow-\hat{p}_{z} & \hat{Q}_{2} \Rightarrow-\hat{p}_{x}
\end{array}
$$




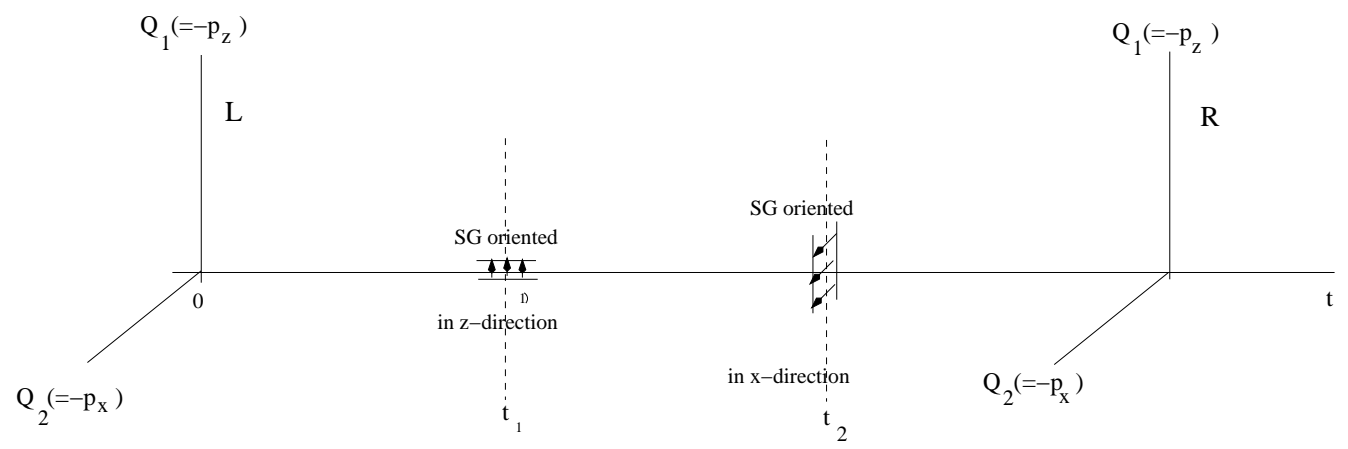

FIGURE 8. Illustration of the measurement of $\left\langle Q_{1} Q_{2}\right\rangle$ and $\left\langle P_{1} Q_{2}\right\rangle$ for a Stern-Gerlach arrangement. The correspondence between the various operators is given in Eqs. (58). Only the axes $Q_{1}$ and $Q_{2}$ are shown. The procedure to measure $\left\langle Q_{1} Q_{2}\right\rangle$ would be: a) send 1 atom from $\mathrm{L}$ to R; b) Measure $Q_{1}=-p_{z}$, $Q_{2}=-p_{x}\left(\left[\hat{Q}_{1}, \hat{Q}_{2}\right]=0\right)$ and construct $Q_{1} Q_{2} ;$ c) Within an ensemble of $\mathscr{N}$ elements, construct $\left\langle Q_{1} Q_{2}\right\rangle=$ $\frac{1}{N} \sum_{i=1}^{\mathscr{N}} Q_{1}^{(i)} Q_{2}^{(i)}$. The procedure to measure $\left\langle P_{1} Q_{2}\right\rangle$ would be to construct $\left\langle P_{1} Q_{2}\right\rangle=\frac{1}{N} \sum_{i=1}^{\mathscr{N}} P_{1}^{(i)} Q_{2}^{(i)}$ in another ensemble of $\mathscr{N}$ elements.

In what follows we examine some properties of the auxiliary functions $W_{b_{m} a_{n}}^{(\hat{B} \leftarrow \hat{A})}\left(\varepsilon_{1}\right)$ and $\tilde{W}_{b_{m} a_{n}}^{(\hat{B} \leftarrow \hat{A})}\left(\varepsilon_{1}\right)$ defined in Eqs. (52) and (56) (for more details, see Refs. [7, 9]).

1) In the strong-coupling limit, $\varepsilon_{1} \rightarrow \infty$, we find

$$
\left.\begin{array}{l}
W_{b_{m} a_{n}}^{(\hat{B} \leftarrow \hat{A})}\left(\varepsilon_{1}\right) \\
\tilde{W}_{b_{m} a_{n}}^{(\hat{B} \leftarrow \hat{A})}\left(\varepsilon_{1}\right)
\end{array}\right\} \rightarrow \mathscr{W}_{b_{m} a_{n}}^{(\hat{B} \leftarrow \hat{A})} \equiv \operatorname{Tr}\left[\rho_{s}^{(0)}\left(\mathbb{P}_{a_{n}} \mathbb{P}_{b_{m}} \mathbb{P}_{a_{n}}\right)\right]
$$

This is the joint probability distribution given by the so-called Wigner's rule [10] (it is real and non-negative; notice its dependence on the order in which the two successive measurements are performed), which is obtained for projective measurements as

$$
\begin{aligned}
P\left(b_{m}, a_{n}\right) & =P\left(b_{m} \mid a_{n}\right) P\left(a_{n}\right)=\left|\left\langle b_{m} \mid a_{n}\right\rangle\right|^{2}\left|\left\langle a_{n} \mid \psi\right\rangle\right|^{2} \\
& =\left\langle\psi\left|\mathbb{P}_{a_{n}} \mathbb{P}_{b_{m}} \mathbb{P}_{a_{n}}\right| \psi\right\rangle .
\end{aligned}
$$

We have assumed a pure state and no degeneracy, and $P\left(b_{m} \mid a_{n}\right)$ denotes a conditional probability.

2) In the weak-coupling limit, $\varepsilon_{1} \rightarrow 0$, we find

$$
\left.\begin{array}{l}
W_{b_{m} a_{n}}^{(\hat{B} \leftarrow \hat{A})}\left(\varepsilon_{1}\right) \\
\tilde{W}_{b_{m} a_{n}}^{(\hat{B} \leftarrow \hat{A})}\left(\varepsilon_{1}\right)
\end{array}\right\} \rightarrow \mathscr{K}_{b_{m} a_{n}}^{(\hat{B} \leftarrow \hat{A})} \equiv \operatorname{Tr}\left[\rho_{s}^{(0)}\left(\mathbb{P}_{b_{m}} \mathbb{P}_{a_{n}}\right)\right]
$$

This is the Kirkwood's-Dirac joint quasi-probability, which is complex, in general [11, 22, 23, 24] (see also Ref. [25]). 
In this limit $\varepsilon_{1} \rightarrow 0$, the probes correlation can be written in various forms as follows

$$
\begin{aligned}
\frac{1}{\varepsilon_{1} \varepsilon_{2}}\left\langle\hat{Q}_{1} \hat{Q}_{2}\right\rangle^{(\hat{B} \leftarrow \hat{A})} & =\sum_{n m} a_{n} b_{m} \frac{1}{2} \operatorname{Tr}\left[\rho_{s}^{(0)}\left(\mathbb{P}_{b_{m}} \mathbb{P}_{a_{n}}+\mathbb{P}_{a_{n}} \mathbb{P}_{b_{m}}\right)\right] \\
& =\sum_{n m} a_{n} b_{m} W_{b_{m} a_{n}}^{M H}=\sum_{n m} a_{n} b_{m}\left\langle\hat{S}_{m n}\right\rangle_{0} \\
& =\frac{1}{2} \operatorname{Tr}\left[\rho_{s}^{(0)}(\hat{B} \hat{A}+\hat{A} \hat{B})\right]
\end{aligned}
$$

where

$$
\begin{aligned}
W_{b_{m} a_{n}}^{M H} & =\frac{1}{2} \operatorname{Tr}\left[\rho_{s}^{(0)}\left(\mathbb{P}_{b_{m}} \mathbb{P}_{a_{n}}+\mathbb{P}_{a_{n}} \mathbb{P}_{b_{m}}\right)\right]=\left\langle\hat{S}_{m n}\right\rangle_{0} \\
\hat{S}_{m n} & \equiv \frac{1}{2}\left(\mathbb{P}_{b_{m}} \mathbb{P}_{a_{n}}+\mathbb{P}_{a_{n}} \mathbb{P}_{b_{m}}\right)
\end{aligned}
$$

$W_{b_{m} a_{n}}^{M H}$ is the real part of the Kirkwood quasi-probability distribution [11, 22, 23, 24], also called the "Margenau-Hill (MH) distribution" [26]: it may take negative values, and thus cannot be regarded as a joint probability in the classical sense.

We remark that, if $\left[\mathbb{P}_{b_{m}}, \mathbb{P}_{a_{n}}\right] \neq 0$, then the operator $\hat{S}_{m n}$ of Eq. (62e) has at least one negative e-value. For this pair of variables, i.e., $a_{n}, b_{m}$, and for the particular state of the system which is the eigenstate that gives rise to this negative eigenvalue, the Margenau-Hill distribution of Eq. (62d) is negative, i.e., $W_{b_{m} a_{n}}^{M H}=\left\langle\hat{S}_{m n}\right\rangle_{0}<0$, and the probes correlation $\left\langle\hat{Q}_{1} \hat{Q}_{2}\right\rangle / \varepsilon_{1} \varepsilon_{2}$ may lie outside the range $\left[\left(a_{n} b_{m}\right)_{\text {min }},\left(a_{n} b_{m}\right)_{\text {max }}\right]$ (see Eq. (62b)). We illustrate this point with an example.

Consider a Hilbert space of dimensionality $N=2$, and two operators $\hat{A}$ and $\hat{B}$ having the following eigenvalues and eigenvectors:

$$
\begin{array}{ll}
a_{n}=1,0 ; & |1\rangle=\left[\begin{array}{l}
1 \\
0
\end{array}\right], \quad|0\rangle=\left[\begin{array}{l}
0 \\
1
\end{array}\right], \\
b_{m}=1,0 ; & \left.\mid 1)=\frac{1}{\sqrt{2}}\left[\begin{array}{l}
1 \\
1
\end{array}\right], \mid 0\right)=\frac{1}{\sqrt{2}}\left[\begin{array}{r}
1 \\
-1
\end{array}\right] .
\end{array}
$$

From Eq. (62b) we have

$$
\frac{1}{\varepsilon_{1} \varepsilon_{2}}\left\langle\hat{Q}_{1} \hat{Q}_{2}\right\rangle^{(\hat{B} \leftarrow \hat{A})}=\sum_{n, m=0}^{1} a_{n} b_{m}\left\langle S_{m n}\right\rangle_{0}=\left\langle S_{11}\right\rangle_{0} .
$$

For the two bases of Eq. (63), we find $S_{11}$ and its eigenvalues as

$$
S_{11}=\frac{1}{4}\left[\begin{array}{ll}
2 & 1 \\
1 & 0
\end{array}\right] \quad \begin{aligned}
& \lambda_{+}=\frac{1}{4}(1+\sqrt{2})>0 \Rightarrow\left|\psi_{+}\right\rangle \\
& \lambda_{-}=\frac{1}{4}(1-\sqrt{2})<0 \Rightarrow\left|\psi_{-}\right\rangle
\end{aligned}
$$

For the state $\left|\psi_{-}\right\rangle$, the position-position correlation of Eq. (64) becomes

$$
\frac{1}{\varepsilon_{1} \varepsilon_{2}}\left\langle\hat{Q}_{1} \hat{Q}_{2}\right\rangle^{(\hat{B} \leftarrow \hat{A})}=\left\langle\psi_{-}\left|S_{11}\right| \psi_{-}\right\rangle=\frac{1}{4}(1-\sqrt{2})<0
$$


which lies outside the interval defined by the possible values 0,1 of the product $s s^{\prime}$.

3) For an intermediate, arbitrary $\varepsilon_{1}, W_{b_{m} a_{n}}^{(\hat{B} \leftarrow \hat{A})}\left(\varepsilon_{1}\right)$ and $\tilde{W}_{b_{m} a_{n}}^{(\hat{B} \leftarrow \hat{A})}\left(\varepsilon_{1}\right)$ can be regarded, as already noted above, as two auxiliary functions, $\mathfrak{R} W_{b_{m} a_{n}}^{(\hat{B} \leftarrow \hat{A})}\left(\varepsilon_{1}\right)$ being taylored for predicting $\left\langle\hat{Q}_{1} \hat{Q}_{2}\right\rangle$, and $\mathfrak{I} \tilde{W}_{b_{m} a_{n}}^{(\hat{B} \leftarrow \hat{A})}\left(\varepsilon_{1}\right)$ for $\left\langle\hat{P}_{1} \hat{Q}_{2}\right\rangle$. In the next section we shall see that for some special $\hat{A}$ 's and $\hat{B}$ 's we can realize the inverse case: from the measurable quantities $\left\langle\hat{Q}_{1} \hat{Q}_{2}\right\rangle$ and $\left\langle\hat{P}_{1} \hat{Q}_{2}\right\rangle$ we can reconstruct $\rho_{s}^{(0)}$.

4) If the projectors $\mathbb{P}_{a_{n}}, \mathbb{P}_{b_{m}}$ appearing in Eqs. (52) and (56) commute, i.e., $\left[\mathbb{P}_{a_{n}}, \mathbb{P}_{b_{m}}\right]=$ $0, \forall n, m$, then we find, for arbitrary $\varepsilon_{1}$

$$
W_{b_{m} a_{n}}^{(\hat{B} \leftarrow \hat{A})}\left(\varepsilon_{1}\right)=\tilde{W}_{b_{m} a_{n}}^{(\hat{B} \leftarrow \hat{A})}\left(\varepsilon_{1}\right)=\operatorname{Tr}\left[\rho_{s}^{(0)}\left(\mathbb{P}_{b_{m}} \mathbb{P}_{a_{n}}\right)\right], \quad \forall \varepsilon_{1}
$$

This result is the standard, real and non-negative, quantum-mechanical definition of the joint probability of $a_{n}$ and $b_{m}$ for commuting observables.

We also find that the correlation of the two probe positions measured in units of $\varepsilon_{1} \varepsilon_{2}$ coincides, for an arbitrary coupling strength $\varepsilon_{1}$, with the standard result for the correlation of the two observables $\hat{A}$ and $\hat{B}$, i.e.,

$$
\frac{1}{\varepsilon_{1} \varepsilon_{2}}\left\langle Q_{1} Q_{2}\right\rangle=\operatorname{Tr}\left[\rho_{s}^{(0)}(\hat{A} \hat{B})\right], \forall \varepsilon_{1}
$$

5) For the particular case in which $\pi_{1}$ is described by a pure Gaussian state, we find (see also Eqs. (42))

$$
\begin{aligned}
\lambda(\beta) & =\tilde{\lambda}(\beta)=g(\beta)=e^{-\frac{\beta^{2}}{8 \sigma_{Q_{1}}^{2}}} \\
W_{b_{m} a_{n}}^{(\hat{B} \leftarrow \hat{A})}\left(\varepsilon_{1}\right) & =\tilde{W}_{b_{m} a_{n}}^{(\hat{B} \leftarrow \hat{A})}\left(\varepsilon_{1}\right) .
\end{aligned}
$$

This is the case studied in Ref. [7].

6) As a particular situation of property 4) above, suppose we measure successively the same observable $\hat{A}$. We thus set $\hat{B}=\hat{A}$ in the formalism. From Eq. (52) we find

$$
\begin{aligned}
W_{a_{\bar{n}} a_{n}}^{(\hat{A} \leftarrow \hat{A})}\left(\varepsilon_{1}\right) & =\sum_{n^{\prime}} \lambda\left(\varepsilon_{1}\left(a_{n}-a_{n^{\prime}}\right)\right) \operatorname{Tr}_{s}\left[\rho_{s}^{(0)}\left(\mathbb{P}_{a_{n^{\prime}}} \mathbb{P}_{a_{\bar{n}}} \mathbb{P}_{a_{n}}\right)\right] \\
& =\operatorname{Tr}_{s}\left(\rho_{s}^{(0)} \mathbb{P}_{a_{n}}\right) \delta_{a_{\bar{n}}, a_{n}} .
\end{aligned}
$$

Eq. (51) then gives

$$
\frac{\left\langle Q_{1} Q_{2}\right\rangle_{f}^{(\hat{A} \leftarrow \hat{A})}}{\varepsilon_{1} \varepsilon_{2}}=\sum_{n, \bar{n}} a_{n} a_{\bar{n}} \mathfrak{N} W_{a_{\bar{n}} a_{n}}^{(\hat{A} \leftarrow \hat{A})}\left(\varepsilon_{1}\right)=\sum_{n} W_{a_{n}}^{(\hat{A})} a_{n}^{2}=\left\langle\hat{A}^{2}\right\rangle_{0} .
$$

For simplicity, we restrict ourselves to the case in which, at $t=0$, the system proper $s$ and the two probes $\pi_{1}, \pi_{2}$ are described by pure states, and

$$
|\Psi\rangle_{0}=|\psi\rangle_{s}^{(0)}|\chi\rangle_{\pi_{1}}^{(0)}|\chi\rangle_{\pi_{2}}^{(0)}
$$


Then, for $t \gg t_{2}$, i.e., after the second interaction, the state vector is given by

$$
\begin{aligned}
|\Psi\rangle_{f} & =\mathrm{e}^{-\frac{i}{\hbar} \varepsilon_{2} \hat{A} \hat{P}_{2}} \mathrm{e}^{-\frac{i}{\hbar} \varepsilon_{1} \hat{A} \hat{P}_{1}}|\Psi\rangle_{0} \\
& =\sum_{n}\left(\mathbb{P}_{a_{n}}|\psi\rangle_{s}^{(0)}\right)\left(\mathrm{e}^{-\frac{i}{\hbar} \varepsilon_{2} a_{n} \hat{P}_{1}}|\chi\rangle_{\pi_{1}}^{(0)}\right)\left(\mathrm{e}^{-\frac{i}{\hbar} \varepsilon_{1} a_{n} \hat{P}_{2}}|\chi\rangle_{\pi_{2}}^{(0)}\right) .
\end{aligned}
$$

The joint probability density (jpd) of the eigenvalues $Q_{1}, Q_{2}$ of the two position operators for times $t>t_{2}$, when the two interactions have ceased to act, is then

$$
\begin{aligned}
p_{f}\left(Q_{1}, Q_{2}\right) & ={ }_{f}\left\langle\Psi\left|\mathbb{P}_{Q_{1}} \mathbb{P}_{Q_{2}}\right| \Psi\right\rangle_{f} \\
& =\sum_{n} W_{a_{n}}^{(\hat{A})}\left|\chi_{\pi_{1}}^{(0)}\left(Q_{1}-\varepsilon_{1} a_{n}\right)\right|^{2}\left|\chi_{\pi_{2}}^{(0)}\left(Q_{2}-\varepsilon_{2} a_{n}\right)\right|^{2} \\
& =\sum_{n} W_{a_{n}}^{(\hat{A})} \frac{\mathrm{e}^{-\frac{\left(Q_{1}-\varepsilon_{1} a_{n}\right)^{2}}{2 \sigma_{Q_{1}}^{2}}}}{\sqrt{2 \pi \sigma_{Q_{1}}^{2}}} \frac{\mathrm{e}^{-\frac{\left(Q_{2}-\varepsilon_{2} a_{n}\right)^{2}}{2 \sigma_{Q_{2}}^{2}}}}{\sqrt{2 \pi \sigma_{Q_{2}}^{2}}} .
\end{aligned}
$$

In Eq. (74c) we have assumed the original pure states for the probes to be Gaussian. Also,

$$
W_{a_{n}}^{(\hat{A})}={ }^{(0)}{ }_{s}\left\langle\psi\left|\hat{\mathbb{P}}_{a_{n}}\right| \psi\right\rangle_{s}^{(0)}
$$

is the Born probability for the value $a_{n}$ in the original system state, and we wrote

$$
\left\langle Q_{1}\left|\mathrm{e}^{-\frac{i}{\hbar} \varepsilon_{1} a_{n} \hat{P}_{1}}\right| \chi\right\rangle_{\pi_{1}}^{(0)}=\chi_{\pi_{1}}^{(0)}\left(Q_{1}-\varepsilon_{1} a_{n}\right)
$$

and similarly for probe $\pi_{2}$.

Clearly, result (71) can be verified from the jpd of Eq. (74c). From this jpd we also obtain

$$
\begin{aligned}
\left\langle\hat{Q}_{i}\right\rangle & =\varepsilon_{i}\langle\hat{A}\rangle_{0}, \quad i=1,2 \\
\left\langle\hat{Q}_{i}^{2}\right\rangle & =\varepsilon_{i}^{2}\left\langle\hat{A}^{2}\right\rangle_{0}+\sigma_{Q_{i}}^{2}, \quad i=1,2
\end{aligned}
$$

It is useful to consider the correlation coefficient between the two probe positions, which is defined as

$$
\begin{aligned}
C\left(Q_{1}, Q_{2}\right) & =\frac{\left\langle Q_{1} Q_{2}\right\rangle-\left\langle Q_{1}\right\rangle\left\langle Q_{2}\right\rangle}{\sqrt{\left[\left\langle Q_{1}^{2}\right\rangle-\left\langle Q_{1}\right\rangle^{2}\right]\left[\left\langle Q_{2}^{2}\right\rangle-\left\langle Q_{2}\right\rangle^{2}\right]}} \\
& =\frac{(\operatorname{var} \hat{A})_{0}}{\sqrt{(\operatorname{var} \hat{A})_{0}+\left(\frac{\sigma_{Q_{1}}}{\varepsilon_{1}}\right)^{2}} \sqrt{(\operatorname{var} \hat{A})_{0}+\left(\frac{\sigma_{Q_{2}}}{\varepsilon_{2}}\right)^{2}}} \\
& \rightarrow 1, \text { as } \sigma_{\mathrm{Q}_{\mathrm{i}}} / \varepsilon_{\mathrm{i}} \rightarrow 0
\end{aligned}
$$

In the second line, (78b), we have used results (77) for a pure Gaussian state of the probes. As a result, in the strong-coupling limit $\sigma_{Q_{i}} / \varepsilon_{i} \rightarrow 0$, the outcomes for the probe 


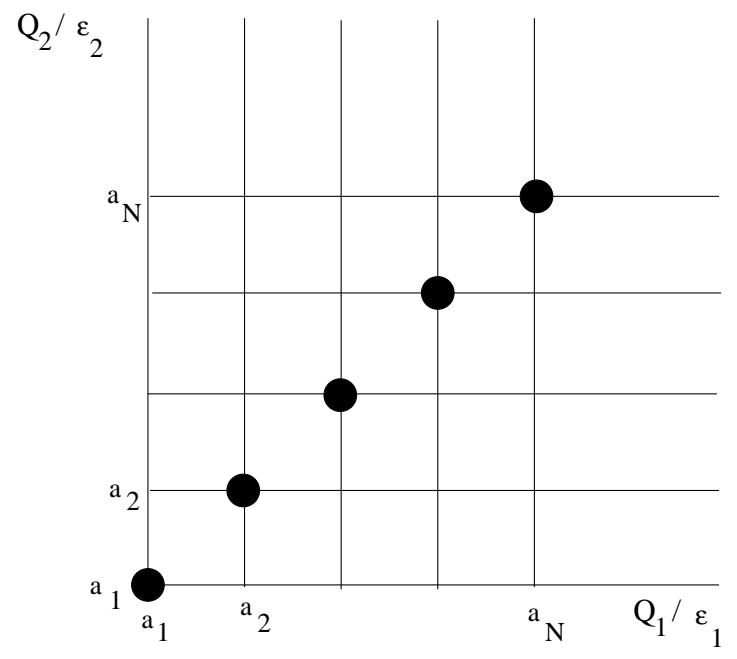

FIGURE 9. Schematic illustration of the jpd of the two probe positions $p_{f}\left(Q_{1}, Q_{2}\right)$ of Eq. (74c), when we measure subsequently the same observable $\hat{A}$. The illustration is for the strong-coupling limit $\sigma_{Q_{i}} / \varepsilon_{i} \ll 1$, in which $Q_{1}$ and $Q_{2}$ are strongly correlated.

position 1 and probe position 2 become completely correlated, which is the result we would have expected. This is illustrated schematically in Fig. 9.

7) Relation of successive measurements to weak values.

The weak value of the observable $\hat{A}$, with pre-selection $|\psi\rangle$ and post-selection $|\phi\rangle$, is defined as $[19,24]$

$$
\begin{aligned}
(\hat{A})_{W} & =\frac{\langle\phi|\hat{A}| \psi\rangle}{\langle\phi \mid \psi\rangle} \\
& =\frac{\langle\psi \mid \phi\rangle\langle\phi|\hat{A}| \psi\rangle}{\langle\psi \mid \phi\rangle\langle\phi \mid \psi\rangle}=\frac{\left\langle\psi\left|\hat{\mathbb{P}}_{\phi} \hat{A}\right| \psi\right\rangle}{\left\langle\psi\left|\hat{\mathbb{P}}_{\phi}\right| \psi\right\rangle} \\
& \Rightarrow \frac{\operatorname{Tr}\left(\hat{\rho}_{s}^{(0)} \hat{\mathbb{P}}_{\phi} \hat{A}\right)}{\operatorname{Tr}\left(\hat{\rho}_{s}^{(0)} \hat{\mathbb{P}}_{\phi}\right)} \\
& =\sum_{n} a_{n} \frac{\left\langle\hat{\mathbb{P}}_{\phi} \hat{\mathbb{P}}_{a_{n}}\right\rangle}{\left\langle\hat{\mathbb{P}}_{\phi}\right\rangle}
\end{aligned}
$$

We see that the weak value can be regarded as the correlation function between the observable $\hat{A}$ and the projector $\hat{\mathbb{P}}_{\phi}[27,28]$. Eq. (79c) gives the generalization of the definition for a state described by a density operator. Eq. (79d) expresses the weak value as the sum over the states of $a_{n}$ times a "complex probability" of $a_{n}$ conditioned by $\phi$.

Consider now a successive measurement experiment in which the two observables are $\hat{A}$ and $\hat{\mathbb{P}}_{\phi}$. The Hamiltonian of Eq. (47) becomes

$$
\hat{H}(t)=\varepsilon_{1} g_{1}(t) \hat{A} \hat{P}_{1}+\varepsilon_{2} g_{2}(t) \hat{\mathbb{P}}_{\phi} \hat{P}_{2}, \quad 0<t_{1}<t_{2} .
$$


One can show $[29,30]$ that the position-position and momentum-position correlation of the two probes are related to the real and imaginary parts of the weak value as

$$
\begin{aligned}
& \lim _{\varepsilon_{1} \rightarrow 0} \frac{\left\langle\hat{Q}_{1} \hat{Q}_{2}\right\rangle^{\left(\hat{\mathbb{P}}_{\phi} \Leftarrow \hat{A}\right)}}{\varepsilon_{1}\left\langle\hat{Q}_{2}\right\rangle^{\left(\hat{\mathbb{P}}_{\phi} \Leftarrow \hat{A}\right)}}=\frac{1}{2} \frac{\left\langle\hat{A} \hat{\mathbb{P}}_{\phi}+\hat{\mathbb{P}}_{\phi} \hat{A}\right\rangle}{\left\langle\hat{\mathbb{P}}_{\phi}\right\rangle}=\Re\left[(\hat{A})_{W}\right], \\
& \lim _{\varepsilon_{1} \rightarrow 0} \frac{\left\langle\hat{P}_{1} \hat{Q}_{2}\right\rangle^{\left(\hat{\mathbb{P}}_{\phi} \Leftarrow \hat{A}\right)}}{\varepsilon_{1}\left\langle\hat{Q}_{2}\right\rangle^{\left(\hat{\mathbb{P}}_{\phi} \Leftarrow \hat{A}\right)}}=\frac{1}{2 \sigma_{Q_{1}}^{2}} \frac{\left\langle\hat{\mathbb{P}}_{\phi} \hat{A}-\hat{A} \hat{\mathbb{P}}_{\phi}\right\rangle}{2 i\left\langle\hat{\mathbb{P}}_{\phi}\right\rangle}=2 \sigma_{P_{1}}^{2} \mathfrak{I}\left[(\hat{A})_{W}\right] .
\end{aligned}
$$

\section{STATE RECONSTRUCTION SCHEME BASED ON SUCCESSIVE MEASUREMENTS}

We now use the above formalism to describe a state tomography scheme. We build on previous work [21,7] to identify a set of observables which, when measured in succession, provide complete information about the state of a quantum system described in an $\mathrm{N}$-dimensional Hilbert space.

For this purpose we consider, in our Hilbert space, two orthonormal bases, whose vectors are denoted by $|k\rangle$ and $\mid \mu)$, respectively, with $k, \mu=1, \ldots, N$. Latin letters will be used to denote the first basis while Greek letters will be used for the second basis. Given $k$, there is only one vector; given $\mu$, there is also only one vector: i.e., we have no degeneracy.

We assume the two bases are mutually non-orthogonal, i.e.,

$$
\langle k| \mu) \neq 0, \quad \forall k, \mu .
$$

Then the two bases have no common eigenvectors. This is illustrated in Fig. 10 for di-

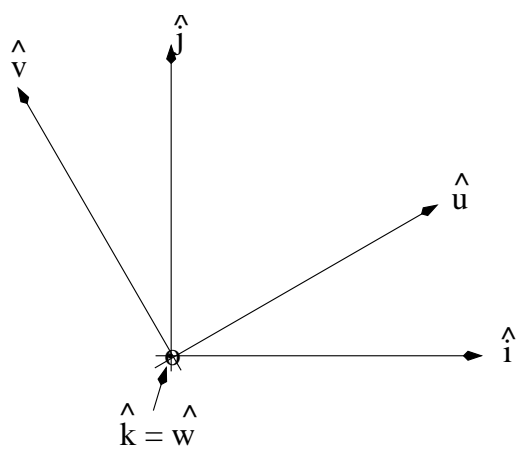

FIGURE 10. Illustration, for $N=2$, of the fact that if the two orthonormal bases have one common eigenvector, they cannot be mutually non-orthogonal.

mensionality $N=3$. The basic vectors of the first basis are $\hat{i}, \hat{j}, \hat{k}$. Those of the second basis are $\hat{u}, \hat{v}, \hat{w}$. Assume they have one common eigenvector: e.g., $\hat{k}=\hat{w}$. Then $\hat{u}, \hat{v} \perp \hat{k}$ and $\hat{i}, \hat{j} \perp \hat{w}$, contradicting the assumption that the two bases have no mutually orthogonal eigenvectors.

We now consider a successive-measurement experiment in which the two observables are the rank-one projectors $\mathbb{P}_{k}$ and $\mathbb{P}_{\mu}$ onto the $k$ - and $\mu$-state of the first and second 
basis, respectively. The Hamiltonian of Eq. (47) becomes

$$
\hat{H}(t)=\varepsilon_{1} g_{1}(t) \mathbb{P}_{k} \hat{P}_{1}+\varepsilon_{2} g_{2}(t) \mathbb{P}_{\mu} \hat{P}_{2} .
$$

The projectors $\mathbb{P}_{k}$ and $\mathbb{P}_{\mu}$ possess two eigenvalues: 0 and 1 . We denote by $\tau$ and $\sigma$ the eigenvalues of $\mathbb{P}_{k}$ and $\mathbb{P}_{\mu}$, respectively, and the corresponding eigenprojectors by $\left(\mathbb{P}_{k}\right)_{\tau}$ and $\left(\mathbb{P}_{\mu}\right)_{\sigma}$. They satisfy relations of the type presented in Eqs. (37).

In the present case, Eq. (51) for the probes position-position correlation function gives

$$
\begin{aligned}
\frac{1}{\varepsilon_{1} \varepsilon_{2}}\left\langle\hat{Q}_{1} \hat{Q}_{2}\right\rangle\left(\mathbb{P}_{\mu} \leftarrow \mathbb{P}_{k}\right) & =\Re \sum_{\tau, \sigma=0}^{1} \tau \sigma W_{\sigma \tau}^{\left(\mathbb{P}_{\mu} \leftarrow \mathbb{P}_{k}\right)}\left(\varepsilon_{1}\right) \\
& =\Re W_{11}^{\left(\mathbb{P}_{\mu} \leftarrow \mathbb{P}_{k}\right)}\left(\varepsilon_{1}\right) .
\end{aligned}
$$

In Eq. (84a), $W_{\sigma \tau}^{\left(\mathbb{P}_{\mu} \leftarrow \mathbb{P}_{k}\right)}\left(\varepsilon_{1}\right)$ is the particular case of the quantity $W_{b_{m} a_{n}}^{(\hat{B} \leftarrow \hat{A})}\left(\varepsilon_{1}\right)$ of Eq. (52) when $\hat{A}, \hat{B}, a_{n}$, and $b_{m}$ are replaced by $\mathbb{P}_{k}, \mathbb{P}_{\mu}, \tau$, and $\sigma$, respectively, i.e.,

$$
W_{\sigma \tau}^{\left(\mathbb{P}_{\mu} \leftarrow \mathbb{P}_{k}\right)}\left(\varepsilon_{1}\right)=\sum_{\tau^{\prime}=0}^{1} \lambda\left(\varepsilon_{1}\left(\tau-\tau^{\prime}\right)\right) \operatorname{Tr}\left[\rho_{s}\left(\mathbb{P}_{k}\right)_{\tau^{\prime}}\left(\mathbb{P}_{\mu}\right)_{\sigma}\left(\mathbb{P}_{k}\right)_{\tau}\right]
$$

and, in particular,

$$
\begin{aligned}
W_{11}^{\left(\mathbb{P}_{\mu} \leftarrow \mathbb{P}_{k}\right)}\left(\varepsilon_{1}\right) & =\operatorname{Tr}\left(\rho_{s}^{(0)} \mathbb{P}_{k} \mathbb{P}_{\mu} \mathbb{P}_{k}\right)+\lambda\left(\varepsilon_{1}\right) \sum_{k^{\prime}(\neq k)} \operatorname{Tr}\left(\rho_{s}^{(0)} \mathbb{P}_{k^{\prime}} \mathbb{P}_{\mu} \mathbb{P}_{k}\right) \\
& =\sum_{k^{\prime}} G_{k k^{\prime}}\left(\varepsilon_{1}\right) \operatorname{Tr}\left(\rho_{s}^{(0)} \mathbb{P}_{k^{\prime}} \mathbb{P}_{\mu} \mathbb{P}_{k}\right),
\end{aligned}
$$

where we have used Eq. (54) and we have defined

$$
G_{k k^{\prime}}\left(\varepsilon_{1}\right)=\delta_{k, k^{\prime}}+\lambda\left(\varepsilon_{1}\right)\left(1-\delta_{k, k^{\prime}}\right) .
$$

An important result is that Eq. (86) can be inverted to obtain $\rho_{s}^{(0)}$, giving $[7,9]$

$$
\left\langle k\left|\rho_{s}^{(0)}\right| k^{\prime}\right\rangle=\sum_{\mu} \frac{W_{11}^{\left(\mathbb{P}_{\mu} \leftarrow \mathbb{P}_{k}\right)}\left(\varepsilon_{1}\right)}{G_{k k^{\prime}}\left(\varepsilon_{1}\right)} \cdot \frac{\left(\mu\left|k^{\prime}\right\rangle\right.}{(\mu|k\rangle},
$$

It is clear that Eq. (88) requires $(\mu|k\rangle \neq 0 \forall \mu$, $k$, i.e., that the two bases must be mutually non-orthogonal. Eq. (88) is the main result of this chapter.

As a consequence, we see that the set of complex quantities $W_{11}^{\left(\mathbb{P}_{\mu} \leftarrow \mathbb{P}_{k}\right)}\left(\varepsilon_{1}\right), \forall k, \mu$, contains all the information about the state of the system $\rho_{s}^{(0)}$. If we could infer $W_{11}^{\left(\mathbb{P}_{\mu} \leftarrow \mathbb{P}_{k}\right)}\left(\varepsilon_{1}\right), \forall k, \mu$, from measurement outcomes, we could reconstruct the full $\rho_{s}^{(0)}$.

Notice that both the real and imaginary parts of the complex quantities $W_{11}^{\left(\mathbb{P}_{\mu} \leftarrow \mathbb{P}_{k}\right)}\left(\varepsilon_{1}\right)$ are needed for tomography. However, from the detected position-position correlations 
$\left\langle\hat{Q}_{1} \hat{Q}_{2}\right\rangle\left(\mathbb{P}_{\mu} \leftarrow \mathbb{P}_{k}\right) / \varepsilon_{1} \varepsilon_{2}$, we directly extract only $\mathfrak{R} W_{11}^{\left(\mathbb{P}_{\mu} \leftarrow \mathbb{P}_{k}\right)}\left(\varepsilon_{1}\right)$, as we see from Eq. (84b). In order to find $\mathfrak{I} W_{11}^{\left(\mathbb{P}_{\mu} \leftarrow \mathbb{P}_{k}\right)}\left(\varepsilon_{1}\right)$, we use the momentum-position correlation. Our aim is to show that the measured quantities, the position-position and momentumposition correlation functions, are informationally complete: that is, one can reconstruct $W_{11}^{\left(\mathbb{P}_{\mu} \leftarrow \mathbb{P}_{k}\right)}$ from these quantities.

We recall, from Eq. (39), the analogous situation in the single-measurement case, where the measurable quantities $\langle\hat{Q}\rangle_{f}^{\left(\hat{\mathbb{P}}_{a_{V}}\right)}$ allow the reconstruction of the diagonal matrix elements of $\rho_{s}^{(0)}$, i.e., $(1 / \varepsilon)\langle\hat{Q}\rangle_{f}^{\left(\hat{\mathbb{P}}_{a_{v}}\right)}=W_{\tau=1}^{\left(\hat{\mathbb{P}}_{a_{v}}\right)}=\left\langle a_{v}\left|\rho_{s}^{(0)}\right| a_{v}\right\rangle$. The extension of this result to the two-probe case is Eq. (88), in conjunction with Eq. (84b) (that relates $\mathfrak{R} W_{11}^{\left(\mathbb{P}_{\mu} \leftarrow \mathbb{P}_{k}\right)}\left(\varepsilon_{1}\right)$ to measurable quantities), and Eq. (96) below (that relates $\mathfrak{I} W_{11}^{\left(\mathbb{P}_{\mu} \leftarrow \mathbb{P}_{k}\right)}\left(\varepsilon_{1}\right)$ to measurable quantities).

In the present case, Eq. (55) for the probes momentum-position correlation function gives

$$
\frac{1}{\varepsilon_{1} \varepsilon_{2}}\left\langle\hat{P}_{1} \hat{Q}_{2}\right\rangle^{\left(\mathbb{P}_{\mu} \leftarrow \mathbb{P}_{k}\right)}=2 \sigma_{P_{1}}^{2} \mathfrak{I} \widetilde{W}_{11}^{\left(\mathbb{P}_{\mu} \leftarrow \mathbb{P}_{k}\right)}\left(\varepsilon_{1}\right)
$$

where

$$
\widetilde{W}_{11}^{\left(\mathbb{P}_{\mu} \leftarrow \mathbb{P}_{k}\right)}\left(\varepsilon_{1}\right)=\operatorname{Tr}\left(\rho_{s}^{(0)} \mathbb{P}_{k} \mathbb{P}_{\mu} \mathbb{P}_{k}\right)+\tilde{\lambda}\left(\varepsilon_{1}\right) \sum_{k^{\prime}(\neq k)} \operatorname{Tr}\left(\rho_{s}^{(0)} \mathbb{P}_{k^{\prime}} \mathbb{P}_{\mu} \mathbb{P}_{k}\right)
$$

Although the function $\widetilde{W}$ is in general not equal to the function $W$ (except when the probes are described by pure Gaussian states, as in Ref. [7]), we now prove that it contains the necessary information to find the imaginary part of $W_{11}^{\left(\mathbb{P}_{\mu} \leftarrow \mathbb{P}_{k}\right)}$, and therefore enables a complete state reconstruction.

If we write

$$
\begin{aligned}
& W_{11}^{\left(\mathbb{P}_{\mu} \leftarrow \mathbb{P}_{k}\right)}=x_{\mu k}+i y_{\mu k}, \\
& \widetilde{W}_{11}^{\left(\mathbb{P}_{\mu} \leftarrow \mathbb{P}_{k}\right)}=\tilde{x}_{\mu k}+i \tilde{y}_{\mu k},
\end{aligned}
$$

the correlation functions, Eqs. (84b) and (89), become

$$
\begin{aligned}
& \frac{\left\langle\hat{Q}_{1} \hat{Q}_{2}\right\rangle^{\left(\mathbb{P}_{\mu} \leftarrow \mathbb{P}_{k}\right)}}{\varepsilon_{1} \varepsilon_{2}}=x_{\mu k}, \\
& \frac{\left\langle\hat{P}_{1} \hat{Q}_{2}\right\rangle\left(\mathbb{P}_{\mu} \leftarrow \mathbb{P}_{k}\right)}{\varepsilon_{1} \varepsilon_{2}}=2 \sigma_{P_{1}}^{2} \tilde{y}_{\mu k} .
\end{aligned}
$$

Our aim is to express $y_{\mu k}$ in terms of the measured quantities of Eqs. (92). We go back to the expressions (86) and (90) for $W_{11}^{\left(\mathbb{P}_{\mu} \leftarrow \mathbb{P}_{k}\right)}\left(\varepsilon_{1}\right)$ and $\widetilde{W}_{11}^{\left(\mathbb{P}_{\mu} \leftarrow \mathbb{P}_{k}\right)}\left(\varepsilon_{1}\right)$. The quantities $\lambda\left(\varepsilon_{1}\right), \tilde{\lambda}\left(\varepsilon_{1}\right)$ are known if the state of the probe $\pi_{1}$ is known (see Eqs. (53) and (57)). We write them as

$$
\begin{aligned}
& \lambda\left(\varepsilon_{1}\right)=\lambda_{r}\left(\varepsilon_{1}\right)+i \lambda_{i}\left(\varepsilon_{1}\right), \\
& \tilde{\lambda}\left(\varepsilon_{1}\right)=\tilde{\lambda}_{r}\left(\varepsilon_{1}\right)+i \tilde{\lambda}_{i}\left(\varepsilon_{1}\right) .
\end{aligned}
$$


On the other hand, the traces appearing in Eqs. (86) and (90) are unknown; we write them as

$$
\begin{aligned}
\operatorname{Tr}\left(\rho_{s}^{(0)} \mathbb{P}_{k} \mathbb{P}_{\mu} \mathbb{P}_{k}\right) & =\left.|\langle k| \mu)\right|^{2}\left\langle k\left|\rho_{s}\right| k\right\rangle=\left.|\langle k| \mu)\right|^{2} \sum_{\mu^{\prime}} x_{\mu^{\prime} k} \\
\sum_{k^{\prime}(\neq k)} \operatorname{Tr}\left(\rho_{s}^{(0)} \mathbb{P}_{k^{\prime}} \mathbb{P}_{\mu} \mathbb{P}_{k}\right) & =r_{\mu k}+i s_{\mu k} .
\end{aligned}
$$

Using Eq. (88), we wrote Eq. (94a) in terms of measured quantities only.

We introduce the definitions (91), (93), (94) in Eqs. (86) and (90), which then give

$$
\begin{aligned}
x_{\mu k} & =\left.|\langle k| \mu)\right|^{2} \sum_{\mu^{\prime}} x_{\mu^{\prime} k}+\lambda_{r} r_{\mu k}-\lambda_{i j} s_{\mu k}, \\
y_{\mu k} & =\lambda_{i} r_{\mu k}+\lambda_{r} s_{\mu k} \\
\tilde{y}_{\mu k} & =\tilde{\lambda}_{i} r_{\mu k}+\tilde{\lambda}_{r} s_{\mu k} .
\end{aligned}
$$

For every pair of indices $\mu, k$ we now have a system of three linear equations in the three unknowns $r_{\mu k}, s_{\mu k}$ and $y_{\mu k}$, which can thus be expressed in terms of the measured quantities $\tilde{y}_{\mu k}$ and the $x_{m k}$ of Eqs. (92). The result for $y_{\mu k}$ is

$$
y_{\mu k}=\frac{\mathfrak{I}\left\{\lambda\left(\varepsilon_{1}\right) \tilde{\lambda}^{*}\left(\varepsilon_{1}\right)\right\}}{\mathfrak{R}\left\{\lambda\left(\varepsilon_{1}\right) \tilde{\lambda}^{*}\left(\varepsilon_{1}\right)\right\}}\left(x_{\mu k}-|\langle k \mid \mu\rangle|^{2} \sum_{\mu^{\prime}} x_{\mu^{\prime} k}\right)+\frac{\left|\lambda\left(\varepsilon_{1}\right)\right|^{2}}{\mathfrak{R}\left\{\lambda\left(\varepsilon_{1}\right) \tilde{\lambda}^{*}\left(\varepsilon_{1}\right)\right\}} \tilde{y}_{\mu k} .
$$

We have thus achieved our goal of expressing $W_{11}^{\left(\mathbb{P}_{\mu} \leftarrow \mathbb{P}_{k}\right)}\left(\varepsilon_{1}\right)$, and hence $\rho_{s}^{(0)}$ of Eq. (88), in terms of the measured correlations of Eqs. (92). This completes our procedure.

It is interesting to examine the strong- and weak-coupling limits of the above procedure. In the strong-coupling limit, $\varepsilon_{1} \rightarrow \infty$, from Eq. (87) we see that $G_{k^{\prime} k}\left(\varepsilon_{1}\right) \rightarrow \delta_{k^{\prime} k}$, and Eq. (88) gives

$$
\left\langle k\left|\rho_{s}^{(0)}\right| k\right\rangle \rightarrow \sum_{\mu} \operatorname{Tr}\left(\rho_{s}^{(0)} \mathbb{P}_{k} \mathbb{P}_{\mu} \mathbb{P}_{k}\right)=\sum_{\mu} \mathscr{W}_{\mu k}^{\left(\mathbb{P}_{\mu} \leftarrow \mathbb{P}_{k}\right)}=\operatorname{Tr}_{s}\left(\rho_{s}^{(0)} \mathbb{P}_{k}\right),
$$

in terms of Wigner's joint probability defined in Eq. (59). Notice that in this limit only the diagonal elements $\rho_{s}^{(0)}$ can be retrieved. This is the limit in which Wigner's formula (59) arises.

In the weak-coupling limit, $\varepsilon_{1} \rightarrow 0$, we have $G_{k^{\prime} k}\left(\varepsilon_{1}\right) \rightarrow 1$, and Eq. (88) gives

$$
\left\langle k\left|\rho_{s}^{(0)}\right| k^{\prime}\right\rangle \rightarrow \sum_{\mu} \operatorname{Tr}_{s}\left(\rho_{s}^{(0)} \mathbb{P}_{\mu} \mathbb{P}_{k}\right) \frac{\left(\mu\left|k^{\prime}\right\rangle\right.}{(\mu|k\rangle}=\sum_{\mu} \mathscr{K}_{\mu k}^{\left(\mathbb{P}_{\mu} \leftarrow \mathbb{P}_{k}\right)} \frac{\left(\mu\left|k^{\prime}\right\rangle\right.}{(\mu|k\rangle}
$$

in terms of Kirkwood's joint quasi-probability defined in Eq. (61). The result (98) was first obtained in Ref. [21].

At first glance it seems that, in a $N$-dimensional Hilbert space, the present scheme for state reconstruction requires the measurement of the $2 N^{2}$ different correlations 
$\left\langle\hat{Q}_{1} \hat{Q}_{2}\right\rangle^{\left(\mathbb{P}_{\mu} \leftarrow \mathbb{P}_{k}\right)}$ and $\left\langle\hat{P}_{1} \hat{Q}_{2}\right\rangle^{\left(\mathbb{P}_{\mu} \leftarrow \mathbb{P}_{k}\right)}$. However, Hermiticity and the unit value of the trace of the density matrix $\rho_{s}^{(0)}$ impose $N^{2}+1$ restrictions among its matrix elements, so that $\rho_{s}^{(0)}$ can be expressed in terms of $N^{2}-1$ independent parameters. These restrictions eventually imply that only $N^{2}-1$ of these correlations are actually independent and thus the measurement of only $N^{2}-1$ correlations is required.

Let us take as an example $N=2$. Labelling the states as $k=0,1$ and $\mu=+,-$, it is enough to measure the correlations

$$
\left\langle\hat{Q}_{1} \hat{Q}_{2}\right\rangle^{\left(\mathbb{P}_{+} \leftarrow \mathbb{P}_{0}\right)},\left\langle\hat{Q}_{1} \hat{Q}_{2}\right\rangle^{\left(\mathbb{P}_{-} \leftarrow \mathbb{P}_{0}\right)} \text {, and }\left\langle\hat{P}_{1} \hat{Q}_{2}\right\rangle^{\left(\mathbb{P}_{-} \leftarrow \mathbb{P}_{0}\right)} \text {. }
$$

In terms of these correlations and using the notation of Eqs. (92), the density matrix elements of our system prior to the mesasurement are given by

$$
\begin{aligned}
\rho_{00}^{(0)} & =x_{+, 0}+x_{-, 0} \\
\rho_{11}^{(0)} & =1-x_{+, 0}-x_{-, 0} \\
\rho_{01}^{(0)} & =\frac{x_{+, 0}-x_{-, 0}-2 i y_{-, 0}}{g\left(\varepsilon_{1}\right)} \\
\rho_{10}^{(0)} & =\frac{x_{+, 0}-x_{-, 0}+2 i y_{-, 0}}{g\left(\varepsilon_{1}\right)} .
\end{aligned}
$$

We have used Gaussian states for the probes, for which $y_{\mu k}=\tilde{y}_{\mu k}$ (see Eqs. (69)).

The relations appearing in Eqs. (86) between $W_{11}^{\left(\mathbb{P}_{\mu} \leftarrow \mathbb{P}_{k}\right)}\left(\varepsilon_{1}\right)$ and $\rho_{s}^{(0)}$ shed light on the the strong- and weak-couping limits of the retrieval scheme described above. We consider again the case $N=2$, for Gaussian states for the probes, and for the case in which the states $|k\rangle, k=0,1$, are eigenstates of the Pauli matrix $\sigma_{k}$ and the states $\mid \mu$ ), $\mu=+,-$, are eigenstates of $\sigma_{x}$.

In the strong-coupling limit $\varepsilon_{1} \rightarrow \infty$,

$$
W_{11}^{\left(\mathbb{P}_{\mu} \leftarrow \mathbb{P}_{k}\right)}\left(\varepsilon_{1}\right) \rightarrow \operatorname{Tr}\left(\rho_{s}^{(0)} \mathbb{P}_{k} \mathbb{P}_{\mu} \mathbb{P}_{k}\right)=\left.|\langle k| \mu)\right|^{2} \rho_{k k}^{(0)},
$$

which, for $N=2$ give

$$
\begin{gathered}
W_{11}^{(+\leftarrow 0)}\left(\varepsilon_{1}\right) \rightarrow \frac{1}{2} \rho_{00}^{(0)}, \quad W_{11}^{(-\leftarrow 0)}\left(\varepsilon_{1}\right) \rightarrow \frac{1}{2} \rho_{00}^{(0)} \\
W_{11}^{(+\leftarrow 1)}\left(\varepsilon_{1}\right) \rightarrow \frac{1}{2} \rho_{11}^{(0)}, W_{11}^{(-\leftarrow 1)}\left(\varepsilon_{1}\right) \rightarrow \frac{1}{2} \rho_{11}^{(0)} .
\end{gathered}
$$

Thus, $W_{11}^{\left(\mathbb{P}_{\mu} \leftarrow \mathbb{P}_{k}\right)}\left(\varepsilon_{1}\right)$ only contains information on the diagonal elements of $\rho_{s}^{(0)}$, so that only $\rho_{00}^{(0)}$ and $\rho_{11}^{(0)}$ can be retrieved.

In the weak-coupling limit $\varepsilon_{1} \rightarrow 0$,

$$
W_{11}^{\left(\mathbb{P}_{\mu} \leftarrow \mathbb{P}_{k}\right)}\left(\varepsilon_{1}\right) \rightarrow \operatorname{Tr}\left(\rho_{s}^{(0)} \mathbb{P}_{\mu} \mathbb{P}_{k}\right)=\mathscr{K}_{\mu k}=\left\langle k\left|\rho_{s}^{(0)}\right| \mu\right)(\mu|k\rangle,
$$


and for $N=2$

$$
\begin{aligned}
& W_{11}^{(+\leftarrow 0)}\left(\varepsilon_{1}\right) \rightarrow \frac{\rho_{00}^{(0)}+\rho_{01}^{(0)}}{2}, \quad W_{11}^{(-\leftarrow 0)}\left(\varepsilon_{1}\right) \rightarrow \frac{\rho_{00}^{(0)}-\rho_{01}^{(0)}}{2} \\
& W_{11}^{(+\leftarrow 1)}\left(\varepsilon_{1}\right) \rightarrow \frac{\rho_{10}^{(0)}+\rho_{11}^{(0)}}{2}, \quad W_{11}^{(-\leftarrow 1)}\left(\varepsilon_{1}\right) \rightarrow \frac{\rho_{10}^{(0)}-\rho_{11}^{(0)}}{2} .
\end{aligned}
$$

We thus see that the four $\rho_{s}^{(0)}$ matrix elements can be retrieved.

In conclusion, to reconstruct a QM state using the successive-measurement scheme, it is better to perform measurements with weak coupling $\varepsilon_{1}$, rather than with strong coupling.

For the case of an arbitrary $\varepsilon_{1}$ we have the relations (100) giving the $\rho_{s}^{(0)}$ matrix elements in terms of the position-position and momentum-position correlations. Recall that $g\left(\varepsilon_{1}\right)=\exp \left(-\frac{\varepsilon_{1}^{2}}{8 \sigma_{Q_{1}}^{2}}\right)$. Even if $g \neq 0$, but $g \ll 1$, a small experimental uncertainty in extracting $\left\langle Q_{1} Q_{2}\right\rangle^{(\mu \leftarrow k)}$ and $\left\langle P_{1} Q_{2}\right\rangle^{(\mu \leftarrow k)}$, which give $W_{11}^{(\mu \leftarrow k)}\left(\varepsilon_{1}\right)=x_{\mu, k}+i y_{\mu, k}$, is divided by a small number $g \ll 1$ when $k \neq k^{\prime}$, and this makes the error in extracting $\rho_{k k^{\prime}}^{(0)}$ large. Again, we see that, in general, it is advantageous to use a weak coupling rather than a strong coupling.

\section{A QUASI-DISTRIBUTION AND A GENERALIZED TRANSFORM OF OBSERVABLES}

Conceptually, one attractive feature of the tomographic approach we have described is that the quantities $W_{11}^{\left(\mathbb{P}_{\mu} \leftarrow \mathbb{P}_{k}\right)}\left(\varepsilon_{1}\right)$ that enter the resonstruction formula, Eq. (88), can be interpreted as a quasi-probability, as we now explain.

Let $\hat{O}$ be an observable associated with an $N$-dimensional quantum system. Using Eq. (88), we can express its expectation value as

$$
\operatorname{Tr}_{s}\left(\hat{\rho}_{s}^{(0)} \hat{O}\right)=\sum_{k k^{\prime}}\left\langle k\left|\rho_{s}^{(0)}\right| k^{\prime}\right\rangle\left\langle k^{\prime}|\hat{O}| k\right\rangle=\sum_{k \mu} W_{11}^{\left(\mathbb{P}_{\mu} \leftarrow \mathbb{P}_{k}\right)}\left(\varepsilon_{1}\right) O\left(\mu, k ; \varepsilon_{1}\right)
$$

where we have defined the "transform" of the operator $\hat{O}$ as

$$
O\left(\mu, k ; \varepsilon_{1}\right)=\sum_{k^{\prime}} \frac{\left(\mu\left|k^{\prime}\right\rangle\right.}{(\mu|k\rangle} \frac{\left\langle k^{\prime}|\hat{O}| k\right\rangle}{G_{k^{\prime} k}\left(\varepsilon_{1}\right)} .
$$

Eqs. (105) have a structure similar to that of a number of transforms found in the literature, that express the quantum mechanical expectation value of an observable in terms of its transform and a quasi-probability distribution.

For example, the Wigner transform of an observable and the Wigner function of a state are defined in the phase space $(q, p)$ of the system, $q$ and $p$ labelling the states of the coordinate and momentum bases, respectively. 
In the present case, the transform (105b) of the observable is defined for the pair of variables $(\mu, k), \mu$ and $k$ labelling the states of each of the two bases. As Eq. (105a) shows, the quantity $W_{11}^{\left(\mathbb{P}_{\mu} \leftarrow \mathbb{P}_{k}\right)}\left(\varepsilon_{1}\right)$ plays the role of a quasi-probability for the system state $\hat{\rho}_{s}^{(0)}$, and is also defined for the pair of variables $(\mu, k)$. It can be thought of as the joint quasi-probability (in general complex [25]) of two non-degenerate observables, the two bases being their respective eigenbases. Since any pair of mutually orthogonal bases can be used, we have a whole family of transforms that can be employed to retrieve the state.

In the literature it has been discussed how Wigner's function can be considered as a representation of a quantum state (see, e.g., Ref. [31], Chs. 3 and 4), in the sense that i) it allows retrieving the density operator, and ii) any quantum-mechanical expectation value can be evaluated from it. Similarly, and for the same reasons, in the present context the quasi-probability $W_{11}^{\left(\mathbb{P}_{\mu} \leftarrow \mathbb{P}_{k}\right)}\left(\varepsilon_{1}\right)$ can also be considered as a representation of a quantum state.

\section{CONCLUSIONS}

In this series of lectures we described how to obtain information on a quantummechanical system, by coupling it to an auxiliary degeree of freedon, or probe, which is then detected by some measuring instrument. The model used in the analysis is the one introduced by von Neumann, in which the interaction of the system proper with the probe is described in a dynamical way.

For single measurements we used the standard von Neumann model, which employs one probe coupled to the system with an arbitrary coupling strength; for successive measurements, we generalized von Neumann's model employing two probes.

In the case of single measurements, we investigated the average, the variance and the full distribution of the probe position after the interaction with the system, and their relation with the properties of the latter. An interesting outcome of the analysis is the reduced density operator of the system which, in the limit of strong coupling between the system and the probe, was shown to reduce to the von-Neumann-Lüders rule, a result which is frequently obtained in a "non-dynamical" way, as a result of non-selective projective measurements.

In the case of successive measurements, we studied how to obtain information on the system by detecting the position-position and momentum-position correlations of the two probes. We saw that the so-called "Wigner's formula", as well as "Kirkwood's quasi-probability distribution", emerge in the strong- and in the weak-coupling limits, respectively, of the above formalism. We investigated the successive measurement of the same observable and showed how, in the strong-coupling limit, the result behaves in the expected manner. The relation of the weak-value theory to successive measurements was briefly mentioned.

Furthermore, we described a quantum state tomography scheme which is applicable to a system described in a Hilbert space of arbitrary finite dimensionality, which is constructed from sequences of two measurements. The scheme consists of measuring the various pairs of projectors onto two bases -which have no mutually orthogonal vectors. 
Finally, we found a generalized transform of the state and the observables based on the notion of successive measurements. The result has a structure similar to that of a number of transforms found in the literature, like the Wigner function, that express the quantum-mechanical expectation value of an observable in terms of its transform and a quasi-probability distribution.

In a recent investigation, the question was posed whether it is possible to find appropriate measurements of the system position and momentum that would allow the reconstruction of the Wigner function of the system state. It was found that the types of measurements needed are successive measurements of projectors associated with position and momentum, of the type envisaged by von Neumann's model which was discussed here. A preliminary account of that investigation can be found in Ref. [32].

\section{ACKNOWLEDGMENTS}

The author acknowledges financial support from Conacyt, Mexico (under Contract No. 79501) and from the Sistema Nacional de Investigadores, Mexico.

\section{REFERENCES}

1. J. von Neumann, Mathematical Foundations of Quantum Mechanics, Princeton Univ. Press, Princeton, N.J., 1955.

2. $\quad$ L. E. Ballentine, Quantum Mechanics. A Modern Development, World Scientific, Singapore, 1999.

3. A. Peres, Quantum Theory: Concepts and Methods, Kluwer Academic Publixhers, Dordrecht, 1995.

4. M. Brune, S. Haroche, J. M. Raimond, L. Davidovich, and N. Zagury, Phys Rev. A 45, 5193 (1992).

5. L. Davidovich, M. Brune, J. M. Raimond, and S. Haroche, Phys Rev. A 53, 1295 (1996).

6. C. Guerlin, J. Bernu, S. Deléglise, C. Sayrin, S. Gleyzes, S. Kuhr, M. Brune, J. M. Raimond, and S. Haroche, Nature 448, 889 (2007).

7. L. M. Johansen and P. A. Mello, Phys. Lett. A 372, 5760-5764 (2008).

8. G. Lüders, Ann. Phys. Lpz. 8, 322 (1951).

9. A. Kalev, and P. A. Mello, J. Phys. A: Math. Theor. 45, 235301 (2012).

10. E. P. Wigner, Am. J. Phys. 31, 6 (1963).

11. J. G. Kirkwood, Phys. Rev. 44, 31 (1933).

12. A. Peres, Phys Rev. D 22, 879 (1980).

13. P. Alstrom, P. Hjorth, and R. Mattuck, Am. J. Phys. 50, 697 (1982).

14. D. E. Platt, Am. J. Phys. 60, 306 (1992).

15. M. O. Scully, W. E. Lamb Jr., and A. Barut, Found. of Phys. 17, 575 (1987).

16. A. Messiah, Quantum Mechanics, North-Holland Publishing Company, Amsterdam, 1986, ISBN .

17. N. Imoto, H. A. Haus, and Y. Yamamoto, Phys Rev. A 32, 2287 (1985).

18. A. Peres, Phys. Rev. D 39, 2943 (1989).

19. D. Z. Aharonov, Y. Albert, and L. Vaidman, Phys. Rev. Lett 60, 1351 (1988).

20. J. S. Bell, and M. Nauenberg, Preludes in Theoretical Physics, A. de Shalit, H. Feshbach, L. van der Hove (Eds.), North Holland, Amsterdam, 1966, p. 279.

21. L. M. Johansen, Phys. Rev. A 76, 012119 (2007).

22. P. A. M. Dirac, Rev. Mod. Phys. 17, 195 (1945).

23. A. M. Steinberg, Phys. Rev. A 52, 32 (1995).

24. L. M. Johansen, and A. Luis, Phys. Rev. A 70, 052115 (2004).

25. R. Feynmann, Quantum Implications; Essays in honour of D. Bohm, B. J. Hiley and F. D. Peat, Editors, Routledge, London and New York, 1987, pp. 235-24, Negative Probability.

26. H. H. Margenau, and R. N., Progr. Theor. Phys. 26, 722 (1961).

27. L. M. Johansen, Phys. Lett. A 322, 298 (2004). 
28. L. M. Johansen, arXiv:Quant-Phys 0804.4379 (2009).

29. L. M. Johansen and P. A. Mello, arXiv:Quant-Phys 0907.5437 (2009).

30. L. M. Johansen (to be published).

31. W. Schleich, Quantum Optics in Phase Space, Wiley-VCH, Berlin, 2001.

32. P. A. Mello, and M. Revzen, ArXiv: Quant-Phys 1307.2877 (2013). 